\title{
Thermal conductivity and interface thermal conductance of thin films in Li ion batteries
}

K. Jagannadham

Materials Science and Engineering

North Carolina State University

Raleigh, NC 27695

e-mail: jag kasichainula@ncsu.edu

Key words: LIPON, LiNiMnO, graphene, thermal conductivity, interface thermal conductance

\begin{abstract}
Laser physical vapor deposition is used to deposit thin films of lithium phosphorous oxynitride in nitrogen and lithium nickel manganese oxide in oxygen ambient on Si substrate. LIPON film is also deposited on LiNiMnO film that is deposited on Si. Graphene films consisting of graphene platelets are deposited on Si substrate from a suspension in isopropyl alcohol. Li-graphene films are obtained after Li adsorption by immersion in $\mathrm{LiCl}$ solution and further drying. Transient thermo reflectance signal is used to determine the cross-plane thermal conductivity of different layers and interface thermal conductance of the interfaces. The results show that LIPON film with lower thermal conductivityis a thermal barrier. The interface thermal conductance between LIPON and $\mathrm{Au}$ or $\mathrm{Si}$ is found to be very low. Thermal conductivity of LiNiMnO is found to be reasonably high so that it is not a barrier to thermal transport. Film with graphene platelets shows a higher value and Li adsorbed graphene film shows a much higher value of cross-plane thermal conductivity. The value of interface thermal conductance between graphene and $\mathrm{Au}$ or $\mathrm{Si}(100)$ substrate is also much lower. The implications of the results for the thermal transport in thin film Li batteries are discussed.
\end{abstract}




\section{Introduction}

The performance of batteries is a function of temperature as the thermal effects [1] include capacity or power fade, self-discharge, thermal runaway and electrical balance in assembly of batteries. Many investigations have been carried out [2-5] on the thermal conductivity of positive and negative electrode materials in large Li-ion batteries used for electric vehicle applications. Fundamental study of lower thermal conductivity of Si nanowires upon Li ion insertion showed phonons are scattered by phonon rattling effect [6]. Thermal conductivity improvement by use of carbon nanotube layers [7] with embedded $\gamma-\mathrm{Fe}_{2} \mathrm{O}_{3}$ particles has been reported. In the present work, we considered the different components of Li-ion thin film batteries and evaluated the thermal conductivity and interface thermal conductance to provide a valuable insight into the critical parameters that control the thermal energy dissipation. The components include thins films of positive electrode of $\mathrm{LiNi}_{0.5} \mathrm{Mn}_{1.5} \mathrm{O}_{4}$ ( $\mathrm{LiNiMnO}$ ), negative electrode of graphene with adsorbed Li ions, and the solid electrolyte of lithium phosphorous oxynitride (LIPON). The choice of the thin films studied in the present work is based upon the high voltage at which LiNiMnO (4.8 V) can be operated for high capacity batteries [8-10], the stability of LIPON upon charge and discharge cycling[11] (5.5 V)and the high capacity of lithium-graphene $[12,13]$ or Li- Si anodes [14] . In order to determine the effect of Li adsorption on graphene platelets, thermal conductivityof graphene thin film consisting of graphene platelets has also been investigatedin the absence of $\mathrm{Li}$. The interface thermal conductance between the thin films and $\mathrm{Si}$ is evaluated as $\mathrm{Si}$ is considered as a high capacity anode material. The importance of microstructure and interface roughness of the components for the thermal energy dissipation in the thin film battery is emphasized.

\section{Experimental Procedure}

\section{1 Preparation of the films}

Thin films of LiNiMnO and LIPON were deposited on Si (100) substrates using laser physical vapor deposition (LPVD). Composite layered film of LIPON on LiNiMnO was also deposited on Si substrate following the same procedure. Target materials of $\mathrm{LiNiMnO}$ and $\mathrm{Li}_{3} \mathrm{PO}_{4}$ were used in the deposition. $\mathrm{Nd}$ - 
YAG laser source in the fourth harmonic $(\lambda=266 \mathrm{~nm})$ with pulse duration of $6 \mathrm{~ns}$, energy per pulse of 210 $\mathrm{mJ}$ and repetition rate of $10 \mathrm{~Hz}$ was used for laser ablation. LiNiMnOfilm was deposited at $550^{\circ} \mathrm{C}$ for $1 \mathrm{hr}$ in oxygen partial pressure of $200 \mathrm{mTorr}(26.64 \mathrm{~Pa})$. LIPON film was deposited at $250^{\circ} \mathrm{C}$ for $1.5 \mathrm{hr}$ in nitrogen partial pressure of $200 \mathrm{mTorr}$ (26.64 Pa). LiNiMnO was deposited first on Si for $1 \mathrm{hr}$ in oxygen and LIPON was deposited on the top for 1.5 hin nitrogen for the composite layered film. The Si substrate was cleaned in acetone followed by native oxide etch in $45 \% \mathrm{HF}$ for $1 \mathrm{~min}$ and rinse in deionized water before loading onto substrate holder in the vacuum chamber. Pre-deposition vacuum of $1.0 \times 10^{-6}$ Torr $\left(133.3 \times 10^{-6} \mathrm{~Pa}\right)$ was achieved by means of a turbo pump backed by amechanical pump. The substrate was heated to the deposition temperature by a resistance heater and monitored with a thermocouple in contact with the substrate holder.

Graphene films were deposited on Si substrate from a suspension of graphene platelets in isopropyl alcohol that was prepared separately. The details of graphene synthesis were described at length in our previous work [15]. Si substrates prepared using the procedure described above were used. Graphene platelet suspension in isopropyl alcohol was collected through a $3 \mathrm{~mm}$ diameter glass tube and dispersed on the Si substrate. The deposition was continued until the thickness of the film was close to $4 \mu \mathrm{m}$. The graphene film was smoothened with a sharp straight edge and dried. The film was not pressed.

Graphene films were lithiated by sonicating in $\mathrm{LiCl}$ solution [16]. LiCl solution was prepared by reacting $\mathrm{Li}$ metal with $10 \mathrm{~mL}$ of concentrated $\mathrm{HCl}$. The addition of $\mathrm{Li}$ foil to the solution mixture was continued until no further reaction of Li was observed. Graphene platelets prepared by drying from the alcohol suspension were dispersed in $10 \mathrm{~mL} \mathrm{LiCl}$ solution and sonicated for $24 \mathrm{hr}$ at low power. Sonication was repeated three times withseparation of $24 \mathrm{hr}$. The pasteof graphene platelets in $\mathrm{LiCl}$ was collected and dispersed on Si substrate to achieve the desired thickness. The film was smoothened with a sharp and straight edge and dried on a hot plate kept at $150^{\circ} \mathrm{C}$ for several hours to remove the $\mathrm{Cl}$ and was 
subsequently retained in a desiccator. X-ray diffraction from the film showed the elimination of hygroscopic LiCl upon repeated drying. Further characterization was carried out using energy dispersive spectrometry (EDS) to determine the absence of $\mathrm{Cl}$ in the film.

The films were characterized by X-ray diffraction for crystallinity, scanning electron microscopy (SEM) for microstructure and morphology, energy dispersive spectroscopy (EDS) for presence of impurities and transient thermoreflectance (TTR) for thermal conductivity and interface thermal conductance.

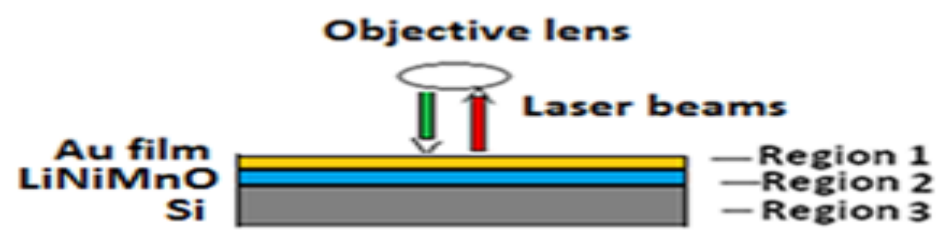

Figure 1. Schematic illustration of geometry of laser incidence on the sample surface. For other samples, LiNiMnO is replaced by the other films. The two laser beams were focused to the same point although shown separate for clarity.

\subsection{Measurement of thermal transport properties}

The value of thermal conductivity and interface thermal conductance was determined by transient thermoreflectance (TTR) measurements. Au transducer film was deposited by LPVD on the surface of the film for purposes of collecting the TTR signal. A schematic of the sample structure used for the TTR signal measurement is shown in figure 1 for LiNiMnO film on Si and the same is valid for the other films. The details of the TTR setup are provided in our previous work [17] and reference [18] and not repeated here for brevity. Briefly, pump laser beam of wave length $532 \mathrm{~nm}$ from second harmonic $\mathrm{Nd}-$ YAG laser at low pulse energy was used to instantaneously heat the substrate at repetition rate of 10 $\mathrm{Hz}$ and the pulse duration was recorded. A continuous laser probe beam of wave length $650 \mathrm{~nm}$ from a laser diode was used to measure the TTR signal. The power of the probe laser was adjusted between 10 to $15 \mathrm{~mW}$ to improve the TTR signal without heating the sample surface. Both the beams were focused 
on to the Au transducer film on the sample through a microscope objective. The reflected light from the sample was collected into a Si photodiode with response time of $1 \mathrm{~ns}$ after filtering the Nd-YAG laser light. The output from the photodiode is amplified through a preamplifier and recorded digitally using an oscilloscope. The oscilloscope signal was triggered using the output from a second Si photodiode that was used to detect the pump beam pulse before it was incident on the sample surface.

\section{Experimental Results}

\subsection{X-ray diffraction}

X-ray diffraction peak profile from LiNiMnO film was collected using a Rigaku instrument in the coupled $\theta-2 \theta$ configuration using $\mathrm{CuK}_{\alpha}$ radiation and the profile is shown in figure 2(a). The peaks are identified from JCPD 32.581 and reference [10]. The LIPON film on Si was amorphous and did not exhibit any peaks. The profile from graphene film on $\mathrm{Si}$ is shown in figure 2(b).

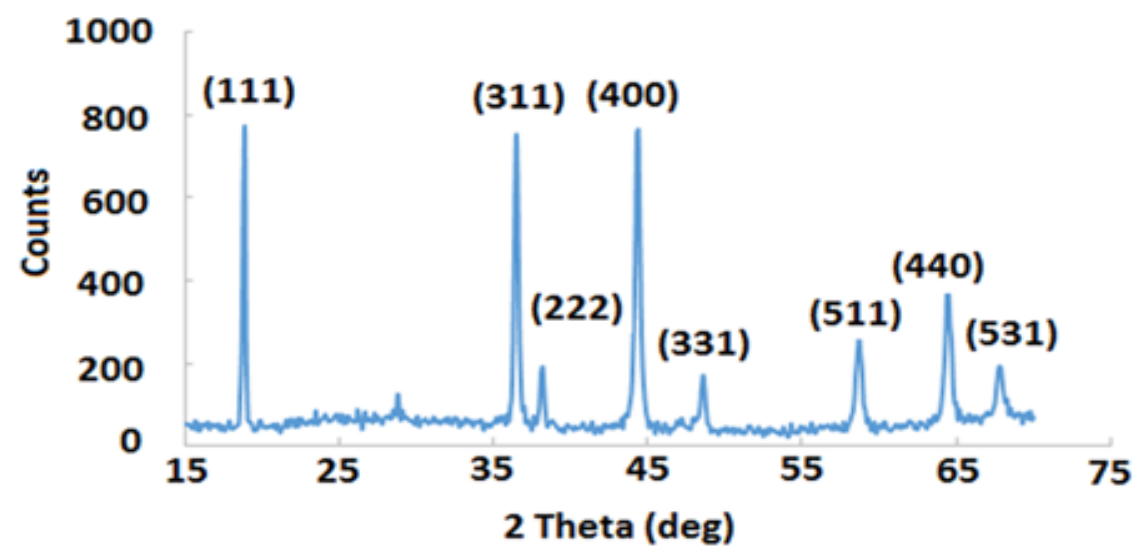

Figure 2(a). X-ray diffraction peak profile obtained from LiNiMnO film deposited on $\mathrm{Si}(100)$ substrate and indexed using JCPD 32-581. The peak at $2 \theta=67.7$ deg was identified to be (531) by others [8] although not listed in the JCPD 32-581. The unidentified peak at 28.6 deg was due to mounting clay on the stage. The Si (004) peak is suppressed by the high atomic number $\mathrm{Ni}$ and $\mathrm{Mn}$ in the film. 


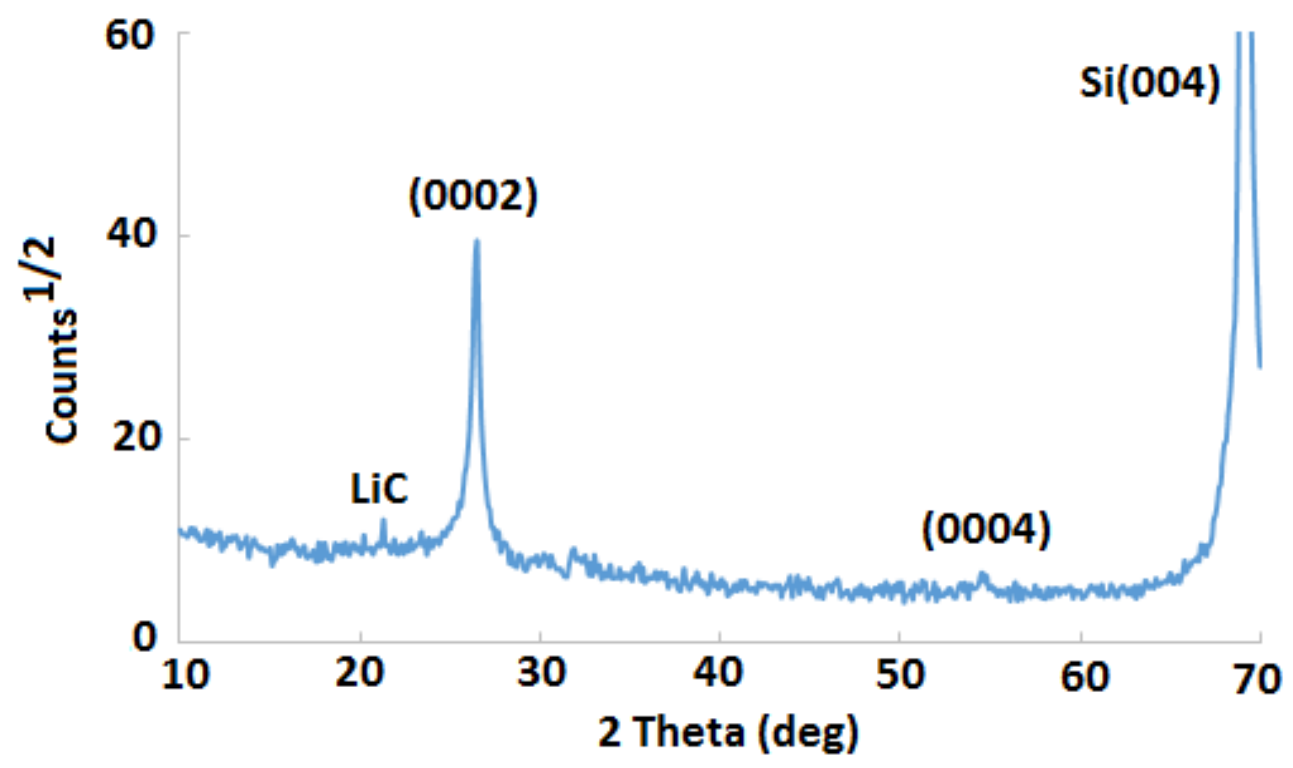

Figure 2(b). X-ray diffraction peak profile obtained from Li-graphene film on Si (100) substrate. The graphite peaks (0002) and (0004) are indexed. The small peak from LiC phase is not strong because both $\mathrm{Li}$ and $\mathrm{C}$ are elements with low atomic number and the fraction of LiC phase in the graphene film is very small.

\subsection{Scanning electron microscopy (SEM) and energy dispersive spectrometry (EDS)}

The samples were examined in the FEI Verios 460L high resolution field emission scanning electron microscope equipped with Oxford EDS detector with high count rate. Both the secondary electron and back scattering detectors were used to collect the images. SEM images of LiNiMnO film shown in figure 3(a) and (b) along with the EDS shown in figure 3(c) indicate that particulates from the laser generated plumeare present. The circular dots are indicative of the molten droplets that are formed during laser ablation but solidified upon reaching the substrate. The absence of impurities in the film is inferred from EDS spectrum presented in figure 3(c). 

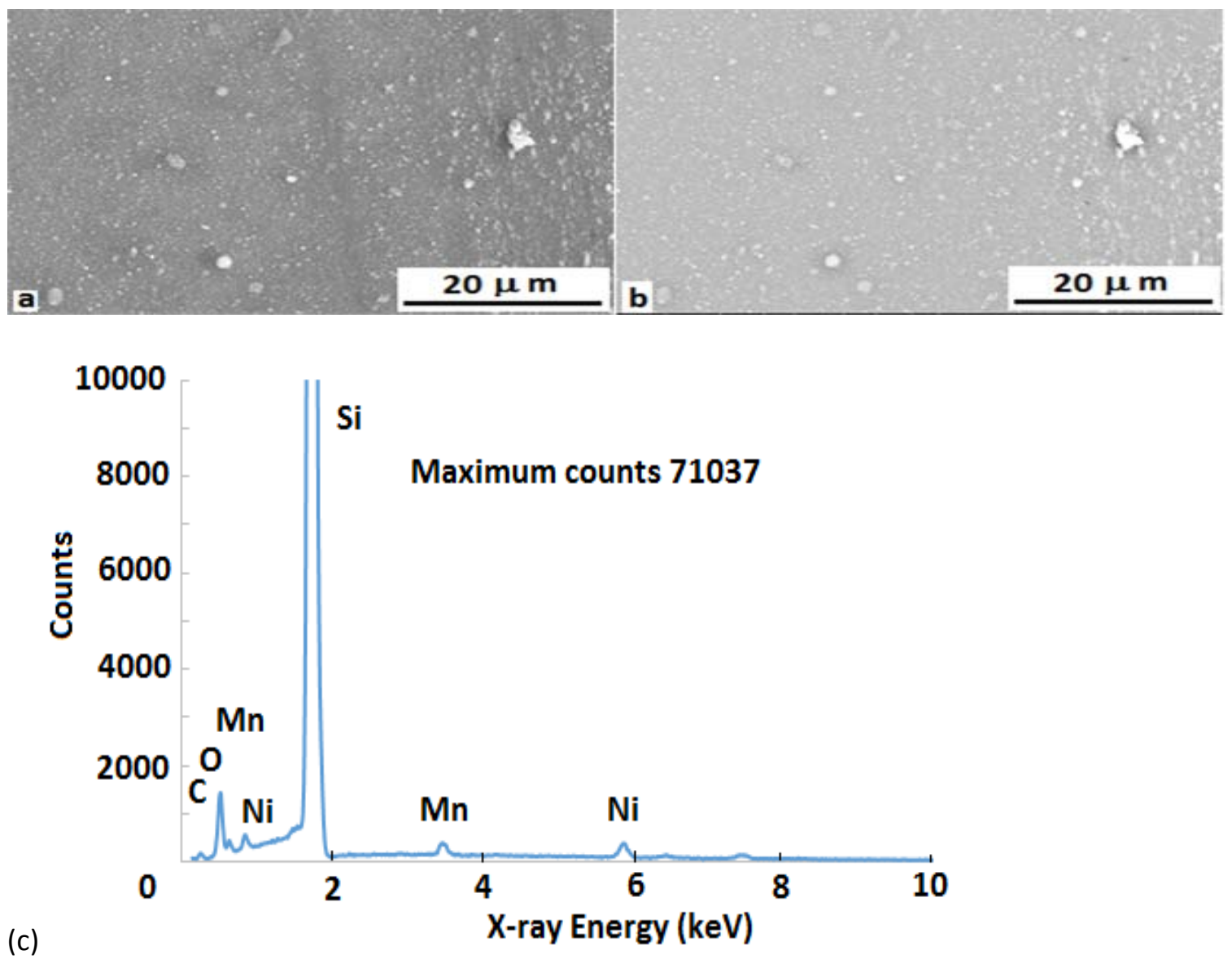

Figure 3. (a) Secondary electron image and (b) backscattering image of the LiNiMnO film on Si substrate deposited by LPVD. The thickness of the film is measured to be $0.4 \mu \mathrm{m}$. (c) Energy dispersive spectrum collected from the LiNiMnO film on Si showing the presence of $\mathrm{Ni}, \mathrm{Mn}$ and $\mathrm{O}$ in the film. The signal from C is thought to arise from hydrocarbons present on the surface in the SEM.

SEM images of LIPON film presented in figure $4(a)$ and (b) along with the EDS shown in figure 4(c) again indicate the presence of particulates in the film. The sintered density of the $\mathrm{Li}_{3} \mathrm{PO}_{4}$ target is low, thus, allowing more particulate ejection from the target. The appearance of the dark particulates in the back scattering image that appear bright in the secondary electron image signifies that the average atomic number of elements in the particulates is low. Thus, the particulates are considered richer in $\mathrm{Li}$ and $\mathrm{O}$ than the film.SEM imagefrom LIPON film deposited on LiNiMnO film on Si is presented in figure 5(a) and (b) from the secondary and backscattering modes, respectively. The sample was coated with Au 
film to obtain a better SEM image. LIPON film on the surface contained laser ablated particulates but otherwise the film is relatively smooth. The EDS spectrum, shown in figure 5(c) contained all the peaks expected including $\mathrm{Ni}, \mathrm{Mn}, \mathrm{O}, \mathrm{Si}$ and $\mathrm{Au}$. The signal from $\mathrm{P}$ that was present between $\mathrm{Si}$ and $\mathrm{Au}$ signal is over shadowed by the strong Au peak but the asymmetry of Au peak indicated the presence of $\mathrm{P}$ in the film.
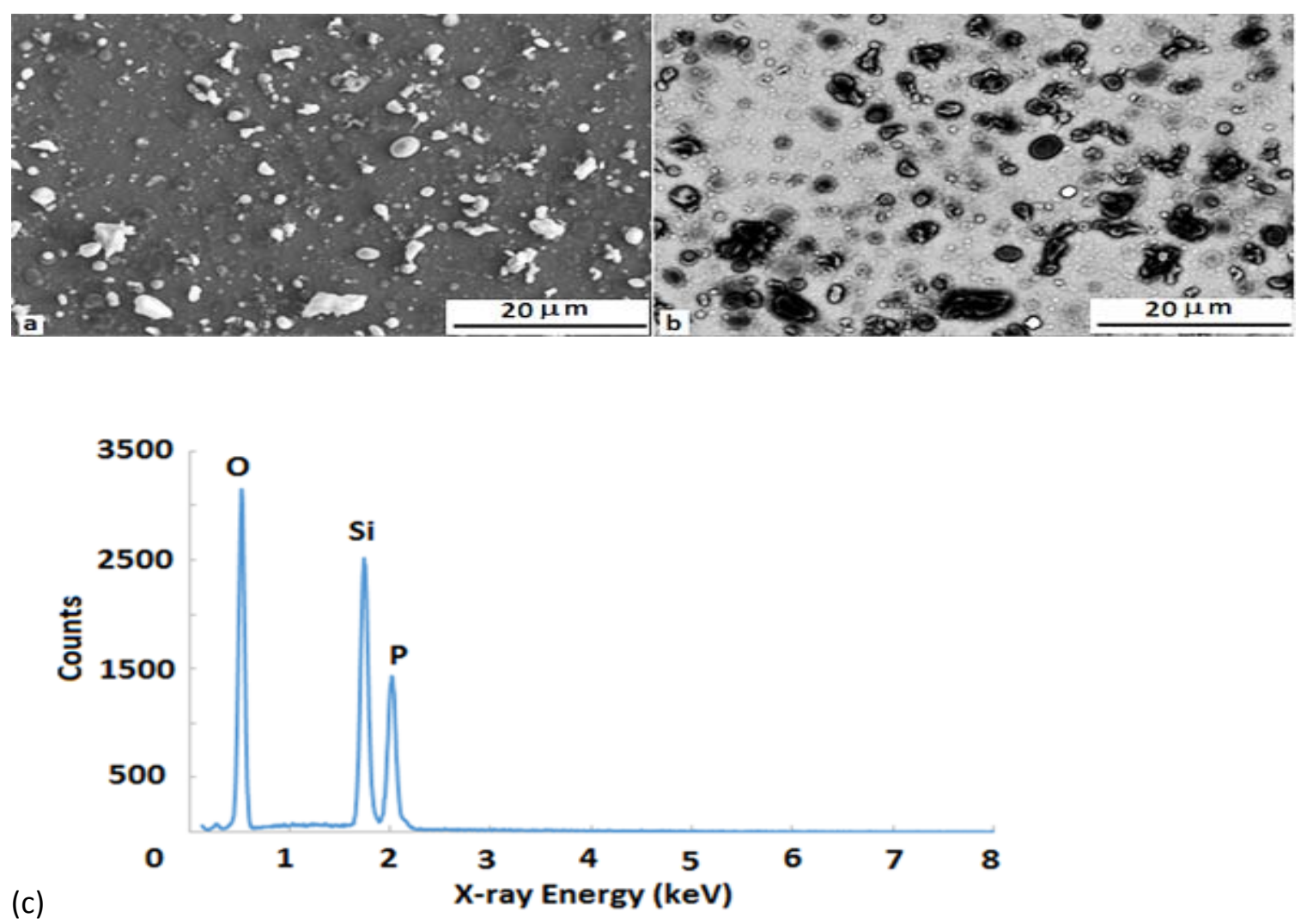

Figure 4. (a) Secondary electron image and (b) backscattering image of the LIPON film on Si substrate deposited by LPVD. The thickness of the film is measured to be $0.4 \mu \mathrm{m}$. (c) Energy dispersive spectrum collected from the LIPON film on Si showing the presence of $\mathrm{P}$ and $\mathrm{O}$ in the film. The signal from $\mathrm{N}$ is very weak. 

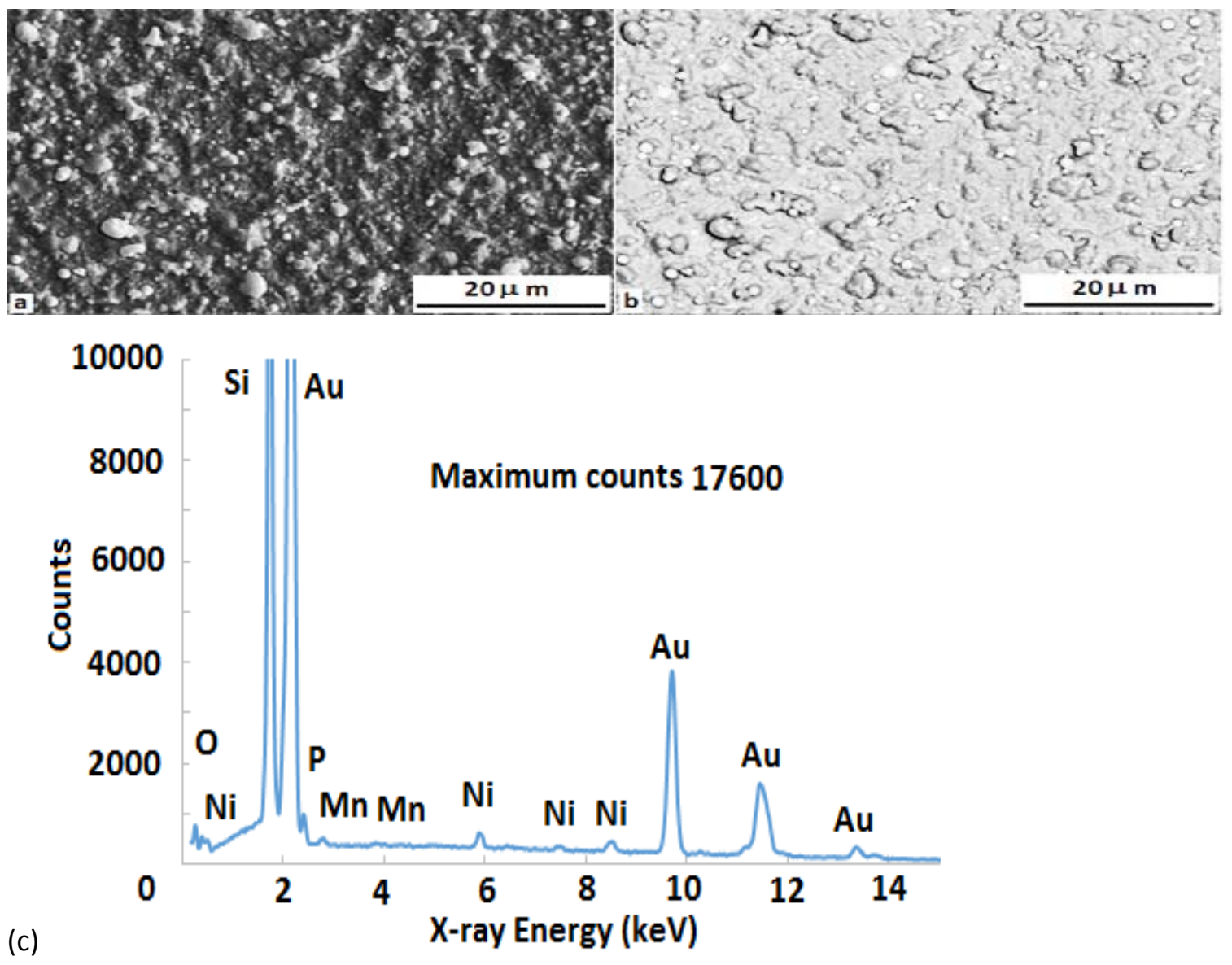

Figure 5. (a) Secondary electron image and (b) backscattering image of the LIPON-LiNiMnO film on Si substrate deposited by LPVD. The thickness of the LIPON film is $0.4 \mu \mathrm{m}$ and that of LiNiMnO film is 0.4 $\mu \mathrm{m}$. (c)Energy dispersive spectrum collected from the LIPON-LiNiMnO film on Si showing the presence of $\mathrm{Ni}, \mathrm{Mn}, \mathrm{P}$ and $\mathrm{O}$ in the film. The signal from $\mathrm{Au}$ is from coating given to obtain better image. Signal from $\mathrm{P}$ is between $\mathrm{Si}$ and Au signals and it is determined by the asymmetry of the Au peak.

SEM images from Li adsorbed graphene film, shown in figure 6(a) and (b), indicate that the surface is not very smooth. Strong C signal from graphene plateletsis present in the EDS spectrum from the film, shown in figure $6(\mathrm{c})$. In addition, $\mathrm{O}$ signalis also present from formation of $\mathrm{Li}_{2} \mathrm{O}$ when the film is heated to drive out the $\mathrm{Cl}$. Au signal arises from the film deposited to improve the image. The weak $\mathrm{Cu}$ signal is thought to arise from an impurity in Li foil that is dissolved in $\mathrm{HCl}$. 

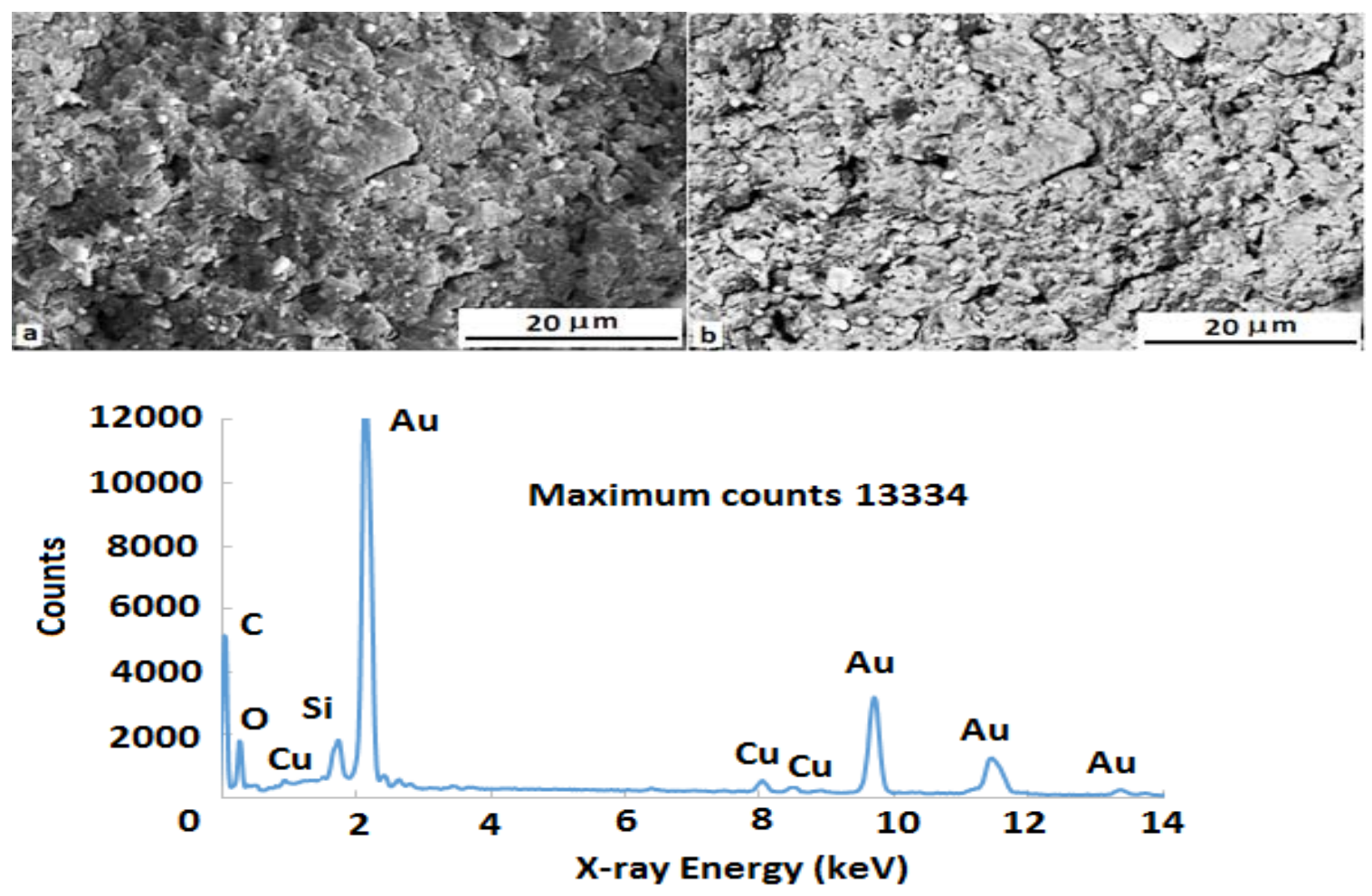

Figure 6. (a) Secondary electron image and (b) backscattering image of the Li-graphene film on Si substrate. Au film of thickness $0.15 \mu \mathrm{m}$ was deposited on the surface to getter a better image of the morphology of the graphene platelets. The thickness of the Li-graphene film is measured to be $4.0 \mu \mathrm{m}$. (c) Energy dispersive spectrum collected from the Li-graphene film on Si showing the presence of C and $\mathrm{O}$ in the film. The small signal from $\mathrm{Cu}$ is considered to arise from the $\mathrm{Li}$ foil that is dissolved in $\mathrm{HCl}$ to form the $\mathrm{LiCl}$. The $\mathrm{O}$ signal is from the $\mathrm{Li}_{2} \mathrm{O}$ formed during heating of the $\mathrm{Li}$-graphene.

\subsection{Transient thermoreflectance (TTR)}

The normalized TTR signal from the different films on Si substrate is shown in figure 7(a) to (e). The wavy experimental result from all the films is a result of surface and interface roughness. The diameter of the pump laser beam is close to $300 \mu \mathrm{m}$ and that of the probe laser beam is $200 \mu \mathrm{m}$. The TTR signal is collected from the smooth Au surface. However, the interface roughness is a function of the surface roughness of the film on which Au film was deposited. In particular, presence of larger size particulates in the LIPON film was responsible for a signal that exhibited higher waviness, asshown in 
figure $7(b)$. The larger value of the laser beam diameter included the effect of surface and interface roughness on the TTR signal.
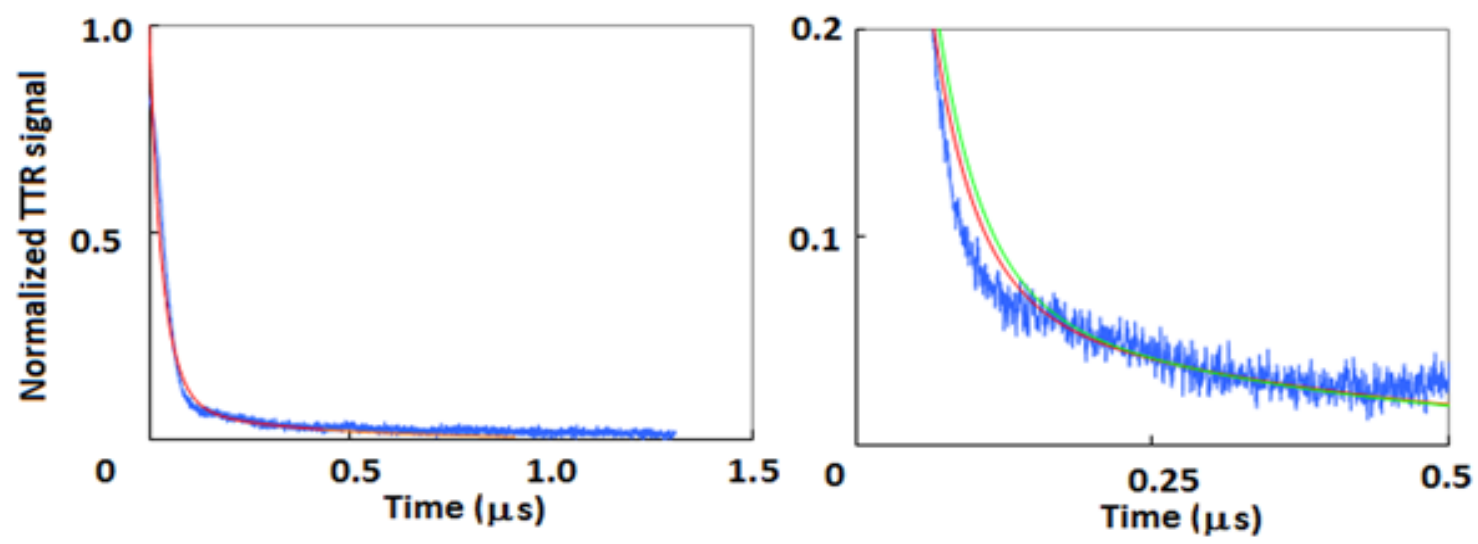

Figure 7(a). Normalized TTR signal from (a) the LiNiMnO film on Si.The blue line is the experimental result. The red line isthe curve fitted result for values of parameters shown in table 1 . The inset on the right shows the details. The green line is obtained for $\mathrm{K}_{2}=30 \mathrm{Wm}^{-1} \mathrm{~K}^{-1}$ and $\mathrm{h}_{23}=150 \mathrm{MWm}^{-2} \mathrm{~K}^{-1}$ but with the same values for other parameters.

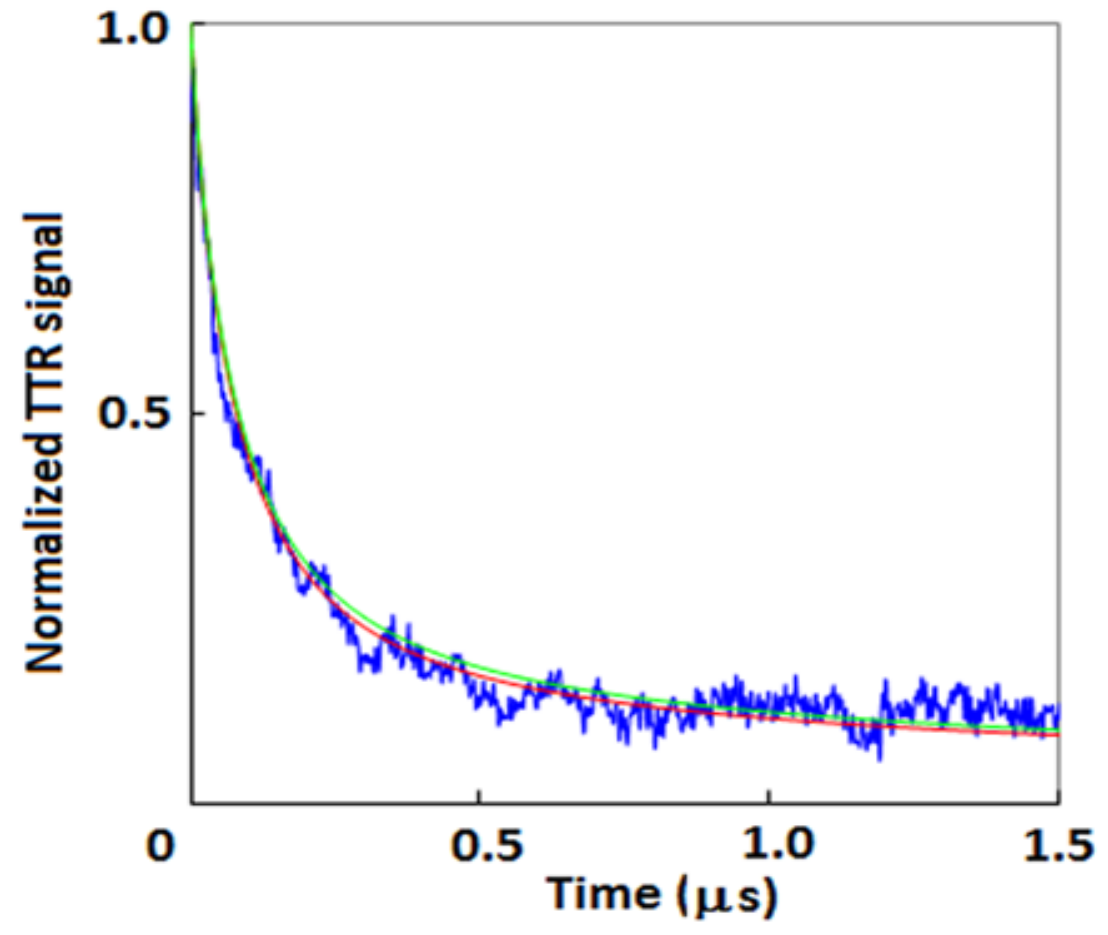


Figure 7(b). Normalized TTR signal fromthe LIPON film on Si. The blue line is the experimental result. The red line is the curve fitted result for values of parameters shown in table 1.The green line is obtained for $\mathrm{K}_{2}=6 \mathrm{Wm}^{-1} \mathrm{~K}^{-1}$ but with the same values for other parameters.

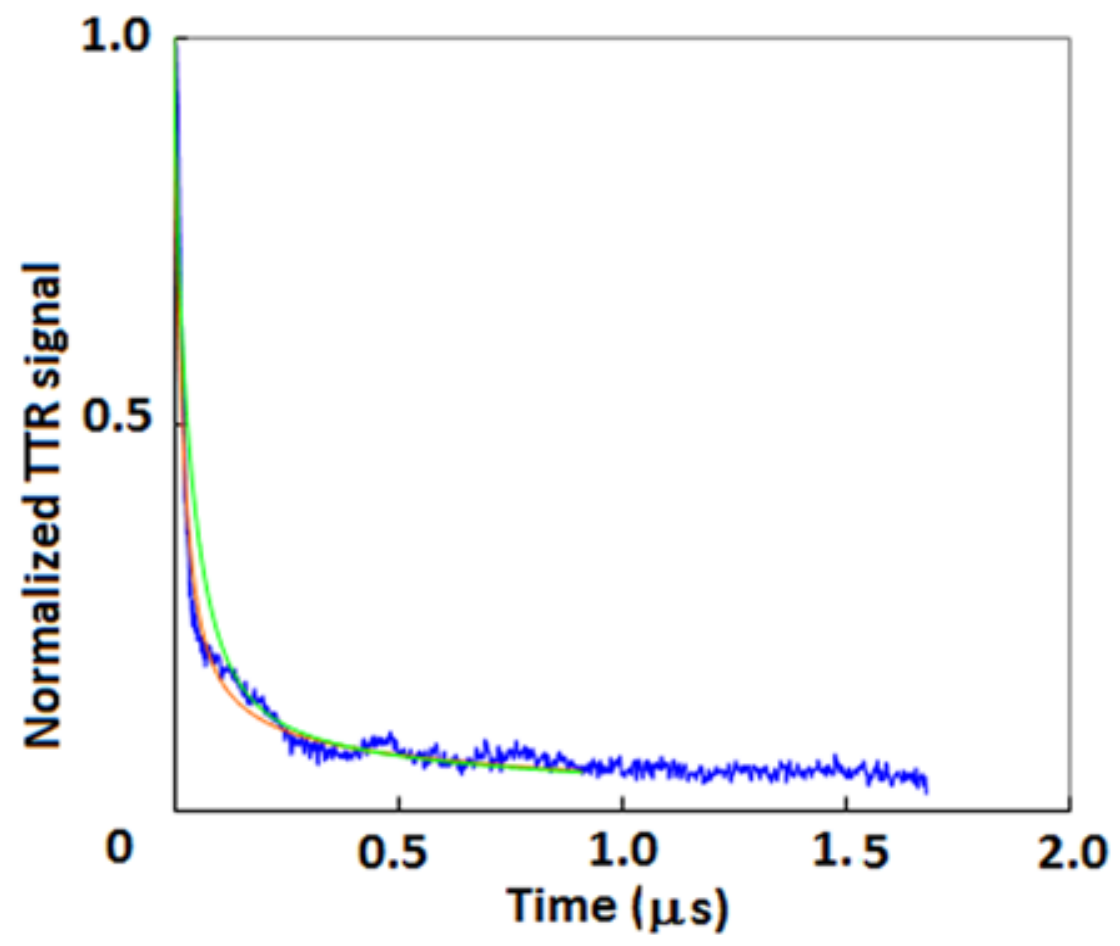

Figure $7(c)$. Normalized TTR signal fromthe LIPON film on LiNiMnO film on Si. The blue line is the experimental result. The red line is the curve fitted result for values of parameters shown in table 1.The green line is obtained for $\mathrm{K}_{2}=5 \mathrm{Wm}^{-1} \mathrm{~K}^{-1}$ but with the same values for other parameters. 


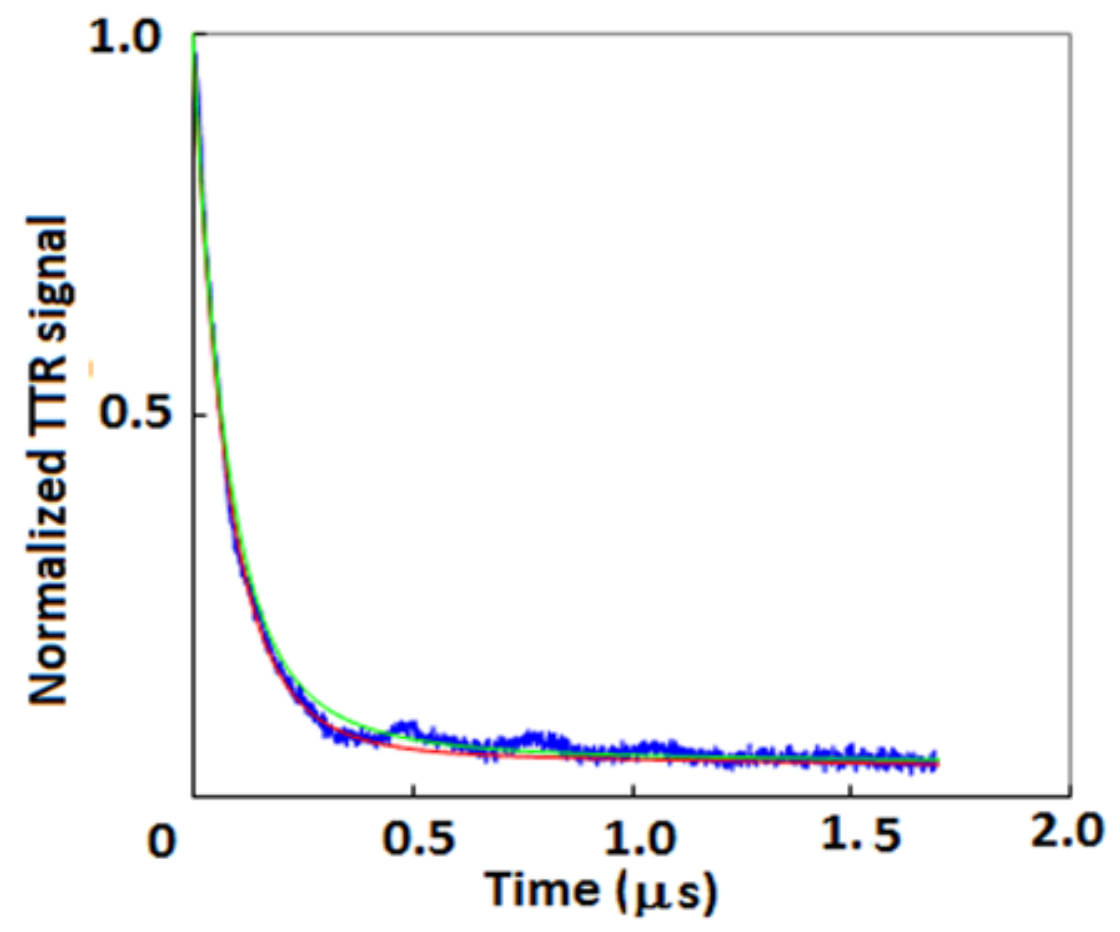

Figure $7(d)$. Normalized TTR signal fromthe Li adsorbed graphene film on Si. The blue line is the experimental result. The red line is the curve fitted result for values of parameters shown in table 1.The green line is obtained for $K_{2}=30 \mathrm{Wm}^{-1} \mathrm{~K}^{-1}, \mathrm{~h}_{12}=\mathrm{h}_{23}=4 \mathrm{MWm}^{-2} \mathrm{~K}^{-1}$ but with the same values for other parameters. 


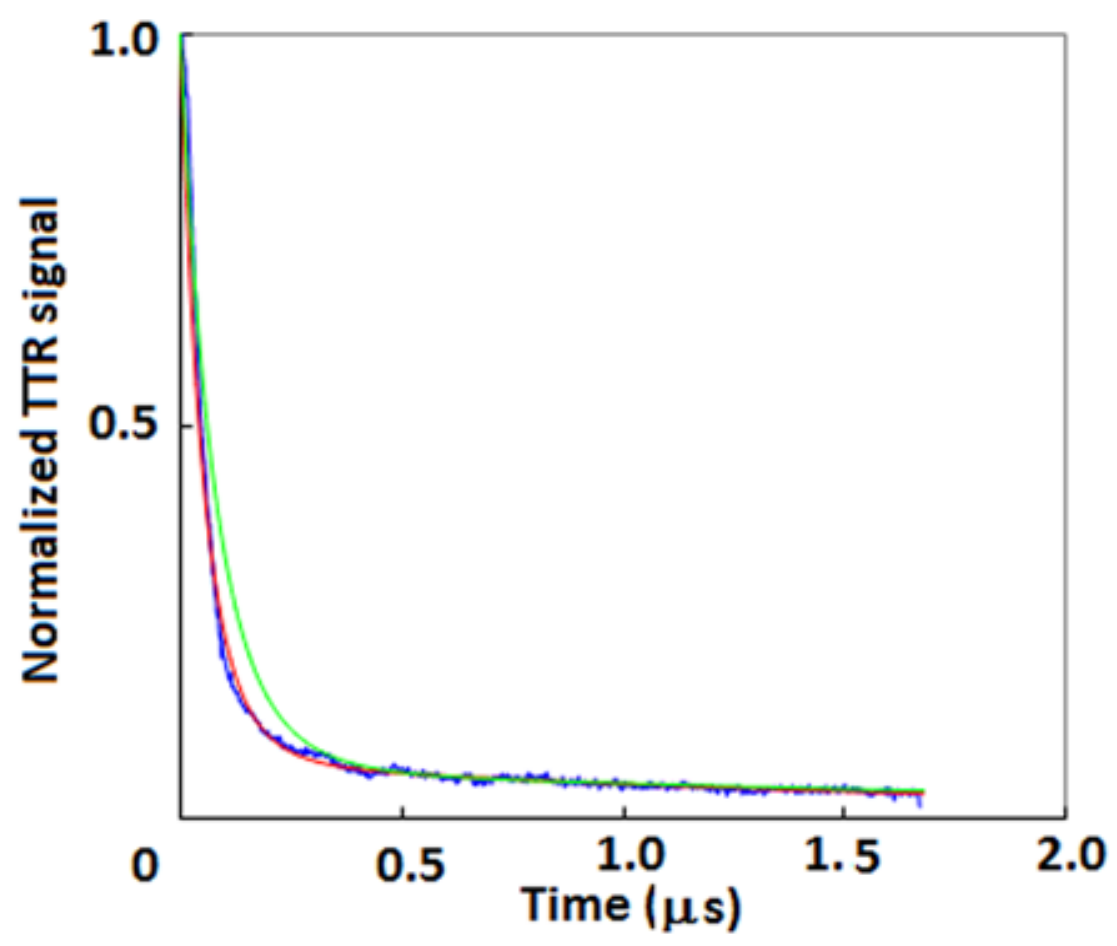

Figure 7(e). Normalized TTR signal from the graphene film on Si. The blue line is the experimental result. The red line is the curve fitted result for values of parameters shown in table 1.The green line is obtained for $\mathrm{K}_{2}=20 \mathrm{Wm}^{-1} \mathrm{~K}^{-1}$ and $\mathrm{h}_{23}=1 \mathrm{MWm}^{-2} \mathrm{~K}^{-1}$ but with the same values for other parameters.

\subsection{Analysis of the TTR signal}

The sample is divided into three regions for purposes of analysis, as shown in figure 1 . Thermal diffusion is one-dimensional $[15,18]$ as the diameter of the incident pump laser beam is much larger than the thermal diffusion depth into the sample. The diffusion equation [17-22] is solved numerically using finite difference method. The boundary conditions include the heat flux at the surface from the incident laser beam and at the interfaces [18]. The interface thermal conductance, $h$, at each interface is determined using flux $\mathrm{J}=-\mathrm{h} d \mathrm{~d}$ where $\mathrm{dT}$ is the temperature difference between the two adjacent regions. Detailed set of equations associated with the boundary conditions at each interface are presented in our previous work [17, 19-22] and reference [18] and not repeated here for brevity. The value of interface thermal conductance at the first interface between Au transducer and the deposited 
film, either LiNiMnO or LIPON or Li-graphene or graphene is $h_{12}$ and that between the film and the Si substrate is $h_{23}$. When the thermal conductivity of the film is very low such as in LIPON, the thermal diffusion depth is very small so that Si substrate and the interface between the film and the substrate are not important in the analysis.

The parameters used in the analysis included the thickness, heat capacity and thermal conductivity of each layer and the two values of interface thermal conductance. The thermal conductivity of the top Au film is measured separately on a sample deposited on Si from the measurement of electrical conductivity and use of Wiedemann-Franz law [23] and found to be $70 \mathrm{Wm}^{-}$ ${ }^{1} \mathrm{~K}^{-1}$. The heat capacity of Auis $2.3 \mathrm{MWm}^{-3} \mathrm{~K}^{-1}$. The thermal diffusion time through the thickness $(0.15 \mu \mathrm{m})$ of the Au transducer film is 0.4 ns. Therefore, thermal diffusion time in Au (region 1) is much smaller than in the film (region 2), $\mathrm{K}_{2}$, present on Si substrate and thus, the results are not significantly affected by the thickness of the Au film. The only parameters that were determined from the analysis were the thermal conductivity of the film (region 2) and the value of the interface thermal conductance of the two interfaces $\left(h_{12}\right.$ and $\left.h_{23}\right)$. The thickness of region $3, t_{3}$, is the value used in the analysis to simulate the results. A value of $t_{3}=8 \mu \mathrm{m}$ is sufficiently large that thermal diffusion occurs well into the substrate. However, $t_{3}$ is smaller when thermal diffusion is reduced. As mentioned previously, the thickness of region 3 was not important when the thermal conductivity of the film (region 2) is very low. The thermal conductivity and the heat capacity of Si was taken to be $150 \mathrm{Wm}^{-1} \mathrm{~K}^{-1}$ and $1.65 \mathrm{MWm}^{-3} \mathrm{~K}^{-1}$, respectively. The heat capacity of graphene platelets is taken to be $1.6 \mathrm{MWm}^{-3} \mathrm{~K}^{-1}[19-22]$.

The normalized TTR signal was curve fitted with the values of the three parameters using least square curve fitting [24] to give $R^{2}$ value close to 0.995 . The two regions of different slope and the middle region of each TTR signal, shown in figures $7(a)$ to (e), were useful to curve fit the TTR signal variation. Results of curve fitting for each sample, shown in in figures 7(a) to (e), contain two curve fitted results in addition to the experimental results. The two curve fitted results are shown to illustrate the 
effect of an important parameter to achieve higher value of $R^{2}$ parameter. The time step was chosen as $0.05 \mathrm{~ns}$ and the distance step was determined to satisfy the stability condition of the numerical solution [24]. The thickness of the film was sufficiently large to separate the two interfaces so that the thermal diffusion time in the film was large and the TTR signal was collected every nanosecond. The results of three parameters used in curve fitting for all the samples are shown table I. The error in the results of analysis that arises from thickness measurements and heat capacity is estimated to be $\sim 10 \%$, as shown in table I.The heat capacity of LiNiMnO is determined from Neumann-Kopp approximation [25] and found to be $3.0 \mathrm{MWm}^{-3} \mathrm{~K}^{-1}$ while that of LIPON is found to be $2.2 \mathrm{MWm}^{-3} \mathrm{~K}^{-1}$. Thickness of region 1 or $\mathrm{Au}$ layer $\left(\mathrm{t}_{1}\right)$ on all samples is $0.15 \mu \mathrm{m}$.

Table I. Thickness and thermal conductivity of different layers used in the analysis. Thickness of the region 2 or the film is $t_{2}$ and thermal conductivity is $K_{2}$. The thickness of region $3\left(t_{3}\right)$ of Si substrate and thermal conductivity $\left(\mathrm{K}_{3}\right)$ are provided. For the sample with composite layer of LIPON on LiNiMnO, region 2 is LIPON and region 3 is LiNiMnO film as the thermal conductivity of LIPON is low and thermal diffusion is limited to the composite layer. The interface thermal conductance between Au film and region 2 is $h_{12}$ and that between region 2 and region 3 is $h_{23}$.

\begin{tabular}{|c|c|c|c|c|c|c|}
\hline Sample & $\begin{array}{c}t_{2} \\
(\mu \mathrm{m}) \quad(W\end{array}$ & $\begin{array}{c}\mathrm{K}_{2} \\
\left.\mathrm{Wm}^{-1} \mathrm{~K}^{-1}\right)\end{array}$ & $\begin{array}{c}t_{3} \\
(\mu \mathrm{m})\end{array}$ & $\begin{array}{c}\mathrm{K}_{3} \\
\left(\mathrm{Wm}^{-1} \mathrm{~K}^{-1}\right)\end{array}$ & $\begin{array}{c}\mathrm{h}_{12} \\
\left(\mathrm{MWm} \mathrm{m}^{-2} \mathrm{~K}^{-1}\right)\end{array}$ & $\begin{array}{c}\mathrm{h}_{23} \\
\left(\mathrm{MWm} \mathrm{m}^{-2} \mathrm{~K}^{-1}\right)\end{array}$ \\
\hline LiNiMnO-Si & 0.4 & $40.0 \pm 5$ & 8.0 & 150 & $8 \pm 1$ & $200 \pm 20$ \\
\hline LIPON-Si & 0.4 & $7.0 \pm 1$ & 8.0 & 150 & $8 \pm 1$ & $10 \pm 2$ \\
\hline LIPON-LiNiMnO & \multicolumn{2}{|l|}{$0.47 .0 \pm 0.8$} & \multicolumn{2}{|c|}{40} & \multicolumn{2}{|c|}{$150 \pm 15$} \\
\hline Graphene-Si & 4.0 & $25.0 \pm 2$ & 8.0 & 150 & $4 \pm 0.5$ & $2 \pm 0.5$ \\
\hline
\end{tabular}


$\begin{array}{lllllll}\text { Li-graphene-Si } & 4.0 & 40.0 \pm 5 & 8.0 & 150 & 6 \pm 1 & 6.5 \pm 1\end{array}$

\section{Discussion}

The LiNiMnO film was crystalline and exhibited all the characteristic peaks in the X-ray diffraction, shown in figure 2(a). SEM images, presented in figure 3(a) and (b),indicate a smooth film consisting of laser generated particulates. EDS results presentedin figure 3(c) containsonly peaks associated with $\mathrm{Ni}$, Mn and O. LIPON film was amorphous and the SEM imagesin figure 4(a) and (b)contained larger particles that showed dark contrast in the backscattering image that indicates higher Li percentage and lower average atomic number. The nitrogen peak in EDS shown in figure 4(c)was weak although the deposition was carried out at $250^{\circ} \mathrm{C}$ in higher nitrogen partial pressure of $200 \mathrm{mTorr}(26.64 \mathrm{~Pa}$ ). The $\mathrm{O}$ and $\mathrm{P}$ peaks were strong. Thus, incorporation of nitrogen into the LIPON structure from nitrogen molecular state may be difficult as the laser energy is not sufficient to fully ionize the $\mathrm{N}_{2}$ gas. Use of $\mathrm{NH}_{3}$ may be favorable to incorporate $\mathrm{N}$ in the amorphous structure, however, it is corrosive to the regular mechanical pump that is used in the present work. SEM image of LIPON film on LiNiMnO filmpresent on Si is shown in figure 5(a) and (b) with EDS presented in figure 5(c). The film is smoother than the LIPON film shown in figure 4. The signal from P in EDS presented in figure 5(c) is over shadowed by the Au peak.

Results of X-ray diffraction, presented in figure 2(b), from the graphene film containing graphene platelets with adsorbed Li from LiCl solution showed the characteristic graphite peaks and a weak peak that is associated with LiC. Thus, the fraction of LiC in the film is small. The surface of the graphene film is not very smooth, as seen in the SEM image in figure 6(a) and (b). The presence of O peak in the EDS, presented in figure $6(\mathrm{c})$, may be a result of heating the film to drive out the $\mathrm{Cl}$ from the $\mathrm{LiCl}$ that is present between the graphene platelets. An external pressure has not been applied to compact the film although a sharp edge is used to smoothen the film. The porous structure at the surface arose from the 
evolution of $\mathrm{Cl}$ from the film. The sample surface is not expected to be smooth like that of vapor deposited film. However, the presence of graphene platelets with large surface areais helpful in the adsorption of $\mathrm{Li}$ from the $\mathrm{LiCl}$ solution.

The emphasis of the present work is to determine the thermal conductivity and interface thermal conductance between films in Li-ion batteries. The measurements using TTR are useful to determine the thermal conductivity in the cross-plane direction and the interface thermal conductance. The thermal conductivity of LiNiMnO film of thickness $0.4 \mu \mathrm{m}$ presented in table 1 , was found to be $\mathrm{K}_{2}=40 \mathrm{Wm}^{-1} \mathrm{~K}^{-1}$ that is reasonably high. Results presented in figure $7(a)$ illustrate that the thermal conductivity of the film, $K_{2}$, and the interface thermal conductance between the film and the Si substrate, $h_{23}$, are important parameters. The film is electrically conducting and therefore both electronic and phonon contributions are present. The electronic contribution arises from the presence of $\mathrm{Li}$ whereas $\mathrm{Ni}$ and $\mathrm{Mn}$ oxides are both known to be semiconducting. Therefore, contribution from electronic contribution will be increased in the discharged state as a result of $\mathrm{Li}$ incorporation and the opposite happens in the charged state. Phonon contribution is expected to be the dominant term in the thermal conductivity. The interface thermal conductance between Au transducer film and LiNiMnO is found to be $8 \mathrm{MWm}^{-2} \mathrm{~K}^{-1}$ that is a low value. The lower value could be due to several reasons but it is primarily associated with the roughness of the film surface as a result of laser generated particulates [26-28]. The thermal resistance at an interface is strongly dependent on the interface roughness as it reduces the thermal contact [28].In addition, thermal conductivity is electron mediated in Au while it is predominantly phonon mediated in LiNiMnO. Therefore, electron-phonon coupling is essential which is known to be low in Au [29]. The interface thermal conductance, $h_{23}$, between LiNiMnO and Si substrate is significantlyhigher with a value of $200 \mathrm{MWm}^{-2} \mathrm{~K}^{-1}$ so that it is not a barrier to thermal transport. The polished surface of Si, the higher crystallinity of LiNiMNO, and the phonon mode thermal conductivity in both the regions is 
responsible for the higher value of $h_{23}$. Thus, the positive electrode material, LiNiMNO, is favorable in terms of thermal energy dissipation.

Results presented in figure 7 (b) illustrate that the thermal conductivity of LIPON film, $K_{2}$, is an important parameter that determines the TTR signal variation. The thermal conductivity of LIPON film of thickness $0.4 \mu \mathrm{m}$, shown in table 1 , is significantly lower at $\mathrm{K}_{2}=7 \mathrm{Wm}^{-1} \mathrm{~K}^{-1}$ and the value of the interface thermal conductance $h_{12}$ between Au and LIPON is also lower at $8 \mathrm{MWm}^{-2} \mathrm{~K}^{-1}$. LIPON is amorphouswith short range or local crystalline order between $\mathrm{P}-\mathrm{O}-\mathrm{N}$ and $\mathrm{P}-\mathrm{O}$ bonds. As a result, the thermal conductivity is not very low as in some amorphous solids such as silica glass [30]. Other estimates [31] of thermal conductivity with a different composition, $\mathrm{Li}_{5.5} \mathrm{PO}_{4.5} \mathrm{~N}_{0.6}$, showed a lower value of $\mathrm{K}_{2}=1.4 \mathrm{Wm}^{-1} \mathrm{~K}^{-}$ ${ }^{1}$ that is much closer to the amorphous limit. The LIPON films deposited in the present work contained much smaller concentration of $\mathrm{N}$ in the film that could be another reason for the slightly higher value of $\mathrm{K}_{2}$. The value of $\mathrm{h}_{23}=10 \mathrm{MWm}^{-2} \mathrm{~K}^{-1}$ is also low as the amorphous LIPON film and the amorphous surface layers of Si containing $\mathrm{N}$ formed during deposition were in contact at the interface. Thus, LIPON filmis found to be a barrier to thermal transport across the positive and negative electrodes. Therefore, thermal energy dissipation on both sides of the electrodes must take place through the metal contact layers.

Results presented in figure 7 (c) illustrate that the thermal conductivity of LIPON is a significant factor in determining the TTR signal variation because the thermal conductivity of LiNiMnO is much higher.The effect of LIPON film in direct contact with LiNiMnO is deduced from the low thermal conductivity of the LIPON film, $\mathrm{K}_{2}=7 \mathrm{Wm}^{-1} \mathrm{~K}^{-1}$ and the thermal conductivity of LiNiMnO, $\mathrm{K}_{3}=40 \mathrm{Wm}^{-1} \mathrm{~K}^{-1}$, presented in table I. The thickness $t_{2}$ of LIPON is $0.4 \mu \mathrm{m}$ while that of LiNiMNO or $t_{3}$ is $0.4 \mu \mathrm{m}$. The interface thermal conductance $h_{12}$ between $A u$ and LIPON is $50 \mathrm{MWm}^{-2} \mathrm{~K}^{-1}$ and that of $h_{23}$ between LIPON and LiNiMnO is $150 \mathrm{MWm}^{-2} \mathrm{~K}^{-1}$. The Si substrate was not needed in the simulation of TTR signal, 
shown in figure $7(\mathrm{c})$,as the thermal diffusion depth was limited to LiNiMnO layer. Theslightly higher value of $h_{12}$ is a result of regions of LIPON film with smaller number of particulates and reduced surface roughness. The much higher value of $h_{23}$ is also associated with the better interfacial contact between LIPON and LiNiMnO and the phonon mediated transport in both regions. The net thermal conductance associated with LIPON and LiNiMnO is determined from $1 / h_{\text {eff }}=t_{2} / K_{2}+1 / h_{23}+t_{3} / K_{3}$ and the resulting value is $h_{\text {eff }}=18 \mathrm{MWm}^{-2} \mathrm{~K}^{-1}$ which is small although $h_{23}$ and $K_{3}$ are reasonably high. These results illustrate that although interface thermal conductance is improved, the lower thermal conductivity of amorphous LIPON is a barrier to thermal transport from positive to negative electrode or vice versa. Thus, the two sides are thermally isolated.

Results presented in figure $7(\mathrm{~d})$ illustrate that the thermal conductivity of Li absorbed graphene, $\mathrm{K}_{2}$, and the interface thermal conductance with the substrate, $h_{23}$, are important parameters. Because the thermal conductivity of the film is relatively higher, the value of $h_{23}$ becomes more important in the thermal dissipation. Similarly, results presented in figure $7(e)$ illustrate that the thermal conductivity of graphene platelets, $K_{2}$, and the interface thermal conductance, $h_{23}$, are important parameters. The thermal conductivity of graphene film with platelets is known tobe high only in the ab plane [32],however, it is low in the C-direction or normal to the ab plane [33]. The thermal conductivity of Li absorbed graphene $\mathrm{K}_{2}=40 \mathrm{Wm}^{-1} \mathrm{~K}^{-1}$, shown in table I, ishigher than that of pure graphene film $\mathrm{K}_{2}=25 \mathrm{Wm}^{-}$ ${ }^{1} \mathrm{~K}^{-1}$ as Li provides a better contact between the platelets. The thermal conductivity of bulk Li is close to $85 \mathrm{Wm}^{-1} \mathrm{~K}^{-1}$ and thus much higher than that of graphene in $\mathrm{C}$-direction which is $35 \mathrm{Wm}^{-1} \mathrm{~K}^{-1}$. Thermal conductivity of Li-graphene composite filmis higher than that of graphene. Therefore, graphene anode exhibits lower heat spreading in the discharged state in the absence of Li. The interface thermal conductance $h_{12}$ between $A u$ and graphene and $h_{23}$ between graphene and Si are both very low, as shown in table I. It is also known that the interface thermal conductance between graphene and other media is low [34] but may be improved by functionalization of graphene [34]. However, the roughness 
of the surface and the poor contact between graphene platelets and $\mathrm{Si}$ is responsible for the lower values of $h_{12}$ and $h_{23}$.

In comparison with other films that are investigated in the present work, graphene platelets show a higher thermal conductivity than the LIPON film but lower than the crystalline LiNiMnO film. The interface thermal conductance of graphene platelets with adsorbed Li is also lower than that of either LIPON or LiNiMnO with Si. Composite films of graphene and Si nano grains that may be used as anodes are also expected to show lower cross-plane thermal conductivity than Si as the interface thermal conductance is lower. The thermal contact is limited between graphene and other films as graphene is inert and only exhibits weak van der Waals bond. The barrier to thermal transport in the films is found to be amorphous LIPON film. Smaller thickness LIPON film is beneficial as long as the film is stable. Alternately, layered composite electrolyte films of $\mathrm{LIPON}$ and $\mathrm{LiLaTiO}_{3}$ (LLT) could improve both ionic conductivity and thermal conductivity and at the same time prevent LLT from chemical instability upon contact with Li ions [35]. The crystalline LLT is expected to have higher thermal conductivity and therefore will improve the value for the composite films above that of the LIPON film provided the interfacial thermal conductance remains higher.

\section{Conclusions}

Thin films of LIPON and LiNiMnO used in Li ion batteries have been deposited by LPVD on Si substrate. The graphene film consisting of Li adsorbed graphene platelets or graphene platelets has been deposited on Si substrate from a suspension. The thermal conductivity of the films in the crossplane was determined by transient thermo reflectance with Au transducer film deposited on the top. The results show that

1. Amorphous LIPON film has much lower thermal conductivity and forms a barrier to thermal transport in the battery. The interface thermal conductance between Au transducer film and LIPON and 
between LIPON and Si was found to be lower as a result of the surface roughness and amorphous structure. However, the interface thermal conductance between LIPON and LiNiMnO is much higher.

2. Thermal conductivity of LiNiMnO is higher. The interface thermal conductance between Au and LiNiMnO is lower because of roughness of the film but that between LiNiMnO and Si is higher. Therefore, LiNiMnO is not a thermal barrier.

3. Thermal conductivity of graphene film withLi adsorbed graphene platelets or with pure graphene platelets is also higher but much smaller compared to the in-plane thermal conductivity. The interface thermal conductance between graphene film and the Au transducer or Si is also lower. Thus, thermal transport is limited by the interface thermal resistance.

\section{Acknowledgements}

This work was performed in part at the Analytical Instrumentation Facility (AIF), which is supported by the State of North Carolina and the National Science Foundation (award number ECCS1542015). The AIF is a member of the North Carolina Research Triangle Nanotechnology Network (RTNN), a site in the National Nanotechnology Coordinated Infrastructure (NNCl). 


\section{References}

1. T. M. Bandhauer, S. Garimella and T. F. Fuller, A critical review of thermal issues in Li-ion batteries, J. Electro. Chem. Soc., 158 (2011) R1-R25

2. H. Maleki, S. A. Hallaj, J. R. Selman, R. B. Dinwiddie and H. Wang, Thermal properties of lithiumion battery and components, J. Electro. Chem. Soc., 146 (1999) 947-954.

3. H. Maleki, J. R. Selman, R. B. Dinwiddie and H. Wang, High thermal conductivity negative electrode material for lithium-ion batteries, J. Power Sources, 94 (2001) 26-35.

4. O. S. Burheim, M. A. Onsrud, J. G. Pharoah, F. Vullum-Bruer and P. J. S. Vie, Thermal conductivity, heat sources and temperature profiles of Li-ion batteries, ECS Trans., 58 (2014) 145-171.

5. V. Vishwakarma and A. Jain, Measurement of in-plane thermal conductivity and heat capacity of separator in Li-ion cells using a transient DC heating method, J. Power Sources, 272 (2014) 378385.

6. W. Xu, G. Zhang and B. Li, Effects of lithium insertion on thermal conductivity of silicon nanowires, Appl. Phys. Lett., 106 (2015) 173901.

7. B. Koo, P. Goli, A. V. Sumant, P. C. D. S. Claro, T. Rajh, C. S. Johnson, A. A. Balandin and E. V. Shevchenko, Toward lithium ion batteries with enhanced thermal conductivity, ACS Nano, 8 (2014)7202-7207.

8. H. Xia, Y.S. Meng, L.Lu, and G. Ceder, Electrochemical properties of nonstoichiometric $\mathrm{LiNi}_{0.5} \mathrm{Mn}_{1.5} \mathrm{O}_{4-\delta}$ thin-film electrodes prepared by pulsed laser deposition, J. Electrochem. Soc., 154 (2007) A737-A743.

9. J. B. Goodenough and K-S. Park, The Li-ion rechargeable battery-A perspective, J. Am. Chem. Soc., 135 (2013) 1167-1176.

10. R. Santhanam and B. Rambabu, Research progress in high voltage spinel $\mathrm{LiNi}_{0.5} \mathrm{Mn}_{1.5} \mathrm{O}_{4}$ material, J. Power Sources, 195 (2010) 5442-5451. 
11. X. Yu, J. B. Bates, G. E. Jellison, Jr., and F. X. Hart, A stable thin-film electrolyte: lithium phosphorous oxynitride, J. Electrochem. Soc., 144 (1997) 524-532.

12. J. Hou, Y. Shao, M. W. Ellis, R. B. Moore and B. Yi, Graphene-based electrochemical energy conversion and storage: fuel cells, supercapacitors and lithium ion batteries, Phys. Chem. Chem. Phys., 13 (2011) 15384-15402.

13. E. Yoo, J. Kim, E. Hosono, H. Zhou, T. Kudo, and I. Honma, Large reversible Li storage of graphene nanosheet families for use in rechargeable lithium ion batteries, Nano Lett., 8 (92008) 22772282.

14. M. T. McDowell, S. W. Lee, W. D. Nix and Y. Cui, $25^{\text {th }}$ Anniversary article: understanding the lithiation of silicon and other alloying anodes for lithium-ion batteries, Adv. Mat., 25 (2013) 4966-4985.

15. H. Zheng and K. Jagannadham, Thermal conductivity and interface thermal conductance in composites of titanium with graphene platelets, J. Heat Trans., 136 (2014) 061301-1-9.

16. L. Mai, B. Hu, W. Chen, Y. Qi, C. Lao, R. Yang, Y. Dai and Z. L. Wang, Lithiated $\mathrm{MoO}_{3}$ nanobelts with greatly improved performance for lithium batteries, Adv. Mater., 19 (2007) 3712-3716.

17. H. Zheng and K. Jagannadham, Interface Thermal conductance between metal films and copper, Met. Mat. Tran. A, 45A (2014) 2480-2486.

18. M. A. Panzer, G. Zhang, D. Mann, X. Hu, E. Pop, H. Dai and K.E. Goodson, J. Heat Trans., 130 (2008)052401-1-9.

19.K. Jaganandham, Effect of interfacial interactions on the thermal conductivity and interfacial thermal conductance in tungsten-graphene layered structure, J. Vac. Sci. Technolog., 32A (2014)051101$1-10$. 
20. K. Jagannadham, Thermal Conductivity and Interface Thermal Conductance in Films of TungstenTungsten Silicide on $\mathrm{Si}_{2}$ IEEE Transactions on Electron Devices, 61 (2014) 1950-1955.

21.H. Zheng and K. Jagannadham, Transient thermoreflectance from graphene composites with matrix of indium and copper, AIP Advances, 3 (2013) 032111-1-13.

22. M. Brown and K. Jagannadham,Thermal Conductivity of MWNT-Epoxy Composites by Transient Thermoreflectance,J. Elect. Mat., 44 (2015) 2624-2630.

23. W. A. Harrison, Solid State Theory, page 263, Dover Publications Inc, New York, 1979.

24. J. B. Scarborough, Numerical mathematical analysis, $4^{\text {th }}$ edition, p. 414 , Oxford University Press, 1958.

25. J. Leitner, P. Vonka, D. Sedmidubsky and P. Svoboda, Application of Neumann-Kopp rule for the estimation of heat capacity of mixed oxides, Thermochim. Acta, 497 (2010) 7-13.

26.P. E. Hopkins, L. M. Phinney, J. R. Serrano and T. E. Beechem, Effects of surface roughness and oxide layer on the thermal boundary conductance at aluminum/silicon interfaces, Proc. $14^{\text {th }}$ Int. heat Transfer Conference, IHTC14, August 8-13, Washington DC, 2010, v. 6, p. 313-319.

27. M. Kazan, A. Bruyant, P. Royer and P. Masri, Thermal conductance of the interfaces between the III-nitride materials and their substrates: Effects of intrinsic material properties and interface conditions, Surf. Sci. Reports, 65 (2010) 111-127.

28. B. N. J. Persson, Thermal interface resistance: cross-over from nanoscale to macroscale,J. Phys. Conden. Matt., 26 (2014) 015009-1-3.

29. A. Birch, W. R. G. Kemp, P. G. Klemens and R. J. Tainsh, Lattice thermal conductivity of some gold alloys, Aust. J. Phys., 12 (1959) 455-465.

30. D. G. Cahill and R. O. Pohl, Thermal conductivity of amorphous solids above the plateau Phys. rev. B, 35 (1987) 4067-4073. 
31. F. Xu,C. Fretigny, D. Fournier, L. Belliard, S. Vincent, B. Perrin, S. Martin, C. Secouard and J.-Y. Duquesne, Lateral heat diffusion investigation of a layered structure: application to the complete thermal characterization of lithium phosphorous oxynitride film, 113 (2013) 244304-1-6.

32. A. A. Balandin, S. Ghosh, W. Bao, I. Calizo, D. Teweldebrahn, F. Miao and C. N. Laue, Superior thermal conductivity of single layer graphene, Nano Lett., 8 (2008) 902-907.

33. P. G. Klemens, Theory of thermal conduction in thin ceramic thin films, Int. J. Thermophysics, 22 (2001) 265-275.

34. E. Hopkins, M. Baraket, E. V. Barnat, T. E. Beechem, S. P. Kearney, J. C. Duda, J. T. Robinson, G. S. Walton, Manipulating thermal conductance at metal-graphene contacts via chemical functionalization, Nano Lett., 12 (2012) 90-595.

35. C. L. Li, B. Zhang and Z. W. Fu, Physical and electrochemical characterization of amorphous lithium lanthanum titanate solid electrolyte thin film fabricated by e-beam evaporation, Thin Solid Films, 515 (2006) 1886-1892. 


\section{List of tables and figures.}

Table I. Thickness and thermal conductivity of different layers used in the analysis. Thickness of the region 2 or the film is $t_{2}$ and thermal conductivity is $K_{2}$. The thickness of region $3\left(t_{3}\right)$ of Si substrate and thermal conductivity $\left(\mathrm{K}_{3}\right)$ are provided. For the sample with composite layer of LIPON on LiNiMnO, region 2 is LIPON and region 3 is LiNiMnO film as the thermal conductivity of LIPON is low and thermal diffusion is limited to the composite layer. The interface thermal conductance between Au film and region 2 is $h_{12}$ and that between region 2 and region 3 is $h_{23}$.

Figure 1. Schematic illustration of geometry of laser incidence on the sample surface. For other samples, LiNiMnO is replaced by the other films. The two laser beams were focused to the same point although shown separate for clarity.

Figure 2(a). X-ray diffraction peak profile obtained from LiNiMnO film deposited on $\mathrm{Si}(100)$ substrate and indexed using JCPD 32-581. The peak at $2 \theta=67.7$ deg was identified to be (531) by others [8] although not listed in the JCPD 32-581. The unidentified peak at 28.6 deg was due to mounting clay on the stage. The Si (004) peak is suppressed by the high atomic number $\mathrm{Ni}$ and $\mathrm{Mn}$ in the film.

Figure 2(b). X-ray diffraction peak profile obtained from Li-graphene film on Si (100) substrate. The graphite peaks (0002) and (0004) are indexed. The small peak from LiC phase is not strong because both $\mathrm{Li}$ and $\mathrm{C}$ are elements with low atomic number and the fraction of LiC phase in the graphene film is very small.

Figure 3. (a) Secondary electron image and (b) backscattering image of the LiNiMnO film on Si substrate deposited by LPVD. The thickness of the film is measured to be $0.4 \mu \mathrm{m}$. (c). Energy dispersive spectrum collected from the LiNiMnO film on $\mathrm{Si}$ showing the presence of $\mathrm{Ni}, \mathrm{Mn}$ and $\mathrm{O}$ in the film. The signal from $\mathrm{C}$ is thought to arise from hydrocarbons present on the surface in the SEM.

Figure 4. (a) Secondary electron image and (b) backscattering image of the LIPON film on Si substrate deposited by LPVD. The thickness of the film is measured to be $0.4 \mu \mathrm{m}$. (c) Energy dispersive spectrum 
collected from the LIPON film on Si showing the presence of $P$ and $O$ in the film. The signal from $N$ is very weak.

Figure 5. (a) Secondary electron image and (b) backscattering image of the LIPON-LiNiMNO film on Si substrate deposited by LPVD. The thickness of the LIPON film is $0.2 \mu \mathrm{m}$ and that of LiNiMnO film is 0.4 $\mu \mathrm{m}$. (c). Energy dispersive spectrum collected from the LIPON-LiNiMnO film on Si showing the presence of $\mathrm{Ni}, \mathrm{Mn}, \mathrm{P}$ and $\mathrm{O}$ in the film. The signal from $\mathrm{Au}$ is from coating given to obtain better image. Signal from $\mathrm{P}$ is between $\mathrm{Si}$ and Au signals and it is determined by the asymmetry of the Au peak.

Figure 6. (a) Secondary electron image and (b) backscattering image of the Li-graphene film on $\mathrm{Si}$ substrate. Au film of thickness $0.15 \mu \mathrm{m}$ was deposited on the surface to getter a better image of the morphology of the graphene platelets. The thickness of the Li-graphene film is measured to be $4.0 \mu \mathrm{m}$. (c). Energy dispersive spectrum collected from the Li-graphene film on Si showing the presence of C and $\mathrm{O}$ in the film. The small signal from $\mathrm{Cu}$ is considered to arise from the Li foil that is dissolved in $\mathrm{HCl}$ to form the $\mathrm{LiCl}$. The $\mathrm{O}$ signal is from the $\mathrm{Li}_{2} \mathrm{O}$ formed during heating of the Li-graphene.

Figure 7(a). Normalized TTR signal from (a) the LiNiMnO film on Si.The blue line is the experimental result. The red line is the curve fitted result for values of parameters shown in table 1 . The green line is obtained for $\mathrm{K}_{2}=30 \mathrm{Wm}^{-1} \mathrm{~K}^{-1}$ and $\mathrm{h}_{23}=150 \mathrm{MWm}^{-2} \mathrm{~K}^{-1}$ but with the same values for other parameters.

Figure 7(b). Normalized TTR signal fromthe LIPON film on Si. The blue line is the experimental result. The red line is the curve fitted result for values of parameters shown in table 1.The green line is obtained for $\mathrm{K}_{2}=6 \mathrm{Wm}^{-1} \mathrm{~K}^{-1}$ but with the same values for other parameters.

Figure $7(\mathrm{c})$. Normalized TTR signal fromthe LIPON film on LiNiMnO film on Si. The blue line is the experimental result. The red line is the curve fitted result for values of parameters shown in table 1.The green line is obtained for $\mathrm{K}_{2}=5 \mathrm{Wm}^{-1} \mathrm{~K}^{-1}$ but with the same values for other parameters.

Figure 7(d). Normalized TTR signal fromthe Li adsorbed graphene film on $\mathrm{Si}$. The blue line is the experimental result. The red line is the curve fitted result for values of parameters shown in table 1.The 
green line is obtained for $K_{2}=30 \mathrm{Wm}^{-1} \mathrm{~K}^{-1}, \mathrm{~h}_{12}=\mathrm{h}_{23}=4 \mathrm{MWm}^{-2} \mathrm{~K}^{-1}$ but with the same values for other parameters.

Figure 7(e). Normalized TTR signal from the graphene film on Si. The blue line is the experimental result. The red line is the curve fitted result for values of parameters shown in table 1.The green line is obtained for $\mathrm{K}_{2}=20 \mathrm{Wm}^{-1} \mathrm{~K}^{-1}$ and $\mathrm{h}_{23}=1 \mathrm{MWm}^{-2} \mathrm{~K}^{-1}$ but with the same values for other parameters. 
Table I. Thickness and thermal conductivity of different layers used in the analysis. Thickness of the region 2 or the film is $t_{2}$ and thermal conductivity is $K_{2}$. The thickness of region $3\left(t_{3}\right)$ of Si substrate and thermal conductivity $\left(\mathrm{K}_{3}\right)$ are provided. For the sample with composite layer of LIPON on LiNiMnO, region 2 is LIPON and region 3 is LiNiMnO film as the thermal conductivity of LIPON is low and thermal diffusion is limited to the composite layer. The interface thermal conductance between Au film and region 2 is $h_{12}$ and that between region 2 and region 3 is $h_{23}$.

$\begin{array}{lcccc}\text { Sample } & \mathrm{t}_{2} & \mathrm{~K}_{2} & \mathrm{t}_{3} & \mathrm{~K}_{3} \mathrm{~h}_{12} \mathrm{~h}_{23} \\ & & & & \\ (\mu \mathrm{m})\left(\mathrm{Wm}^{-1} \mathrm{~K}^{-1}\right) & (\mu \mathrm{m})\left(\mathrm{Wm}^{-1} \mathrm{~K}^{-1}\right) & \left(\mathrm{MWm}^{-2} \mathrm{~K}^{-1}\right) & \left(\mathrm{MWm}^{-2} \mathrm{~K}^{-1}\right)\end{array}$

$\begin{array}{lcccccc}\text { LiNiMnO-Si } & 0.4 & 40.0 \pm 5 & 8.0 & 150 & 8 \pm 1 & 200 \pm 20 \\ \text { LIPON-Si } & 0.4 & 7.0 \pm 1 & 8.0 & 150 & 8 \pm 1 & 10 \pm 2 \\ \text { LIPON-LiNiMnO } & 0.4 & 7.0 \pm 1 & 0.4 & 40 & 50 \pm 5 & 150 \pm 15 \\ \text { Graphene-Si } & 4.0 & 25.0 \pm 2 & 8.0 & 150 & 4 \pm 0.5 & 2 \pm 0.5 \\ \text { Li-graphene-Si } & 4.0 & 40.0 \pm 5 & 8.0 & 150 & 6 \pm 1 & 6.5 \pm 1 \\ & & & & & & \end{array}$




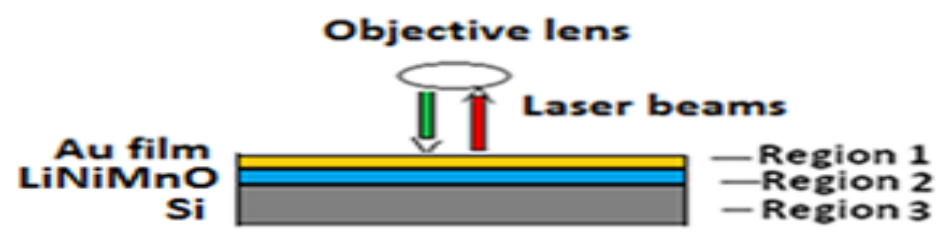

Figure 1. Schematic illustration of geometry of laser incidence on the sample surface. For other samples, LiNiMnO is replaced by the other films. The two laser beams were focused to the same point although shown separate for clarity. 


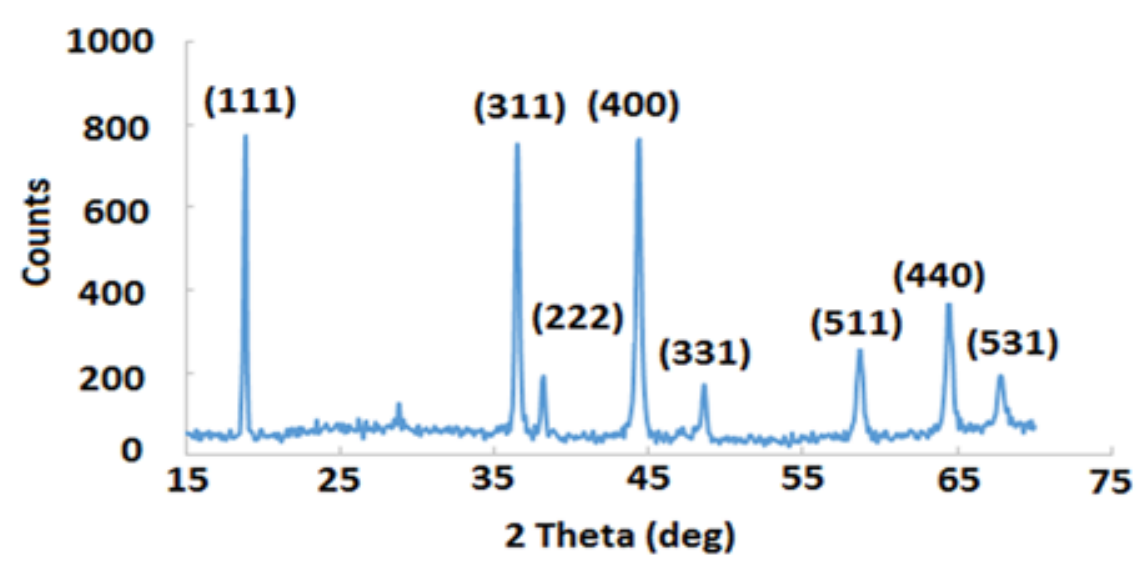

Figure 2(a). X-ray diffraction peak profile obtained from LiNiMnO film deposited on Si(100) substrate and indexed using JCPD 32-581. The peak at $2 \theta=67.7 \mathrm{deg}$ was identified to be (531) by others [8] although not listed in the JCPD 32-581. The unidentified peak at 28.6 deg was due to mounting clay on the stage. The Si (004) peak is suppressed by the high atomic number Ni and $\mathrm{Mn}$ in the film. 


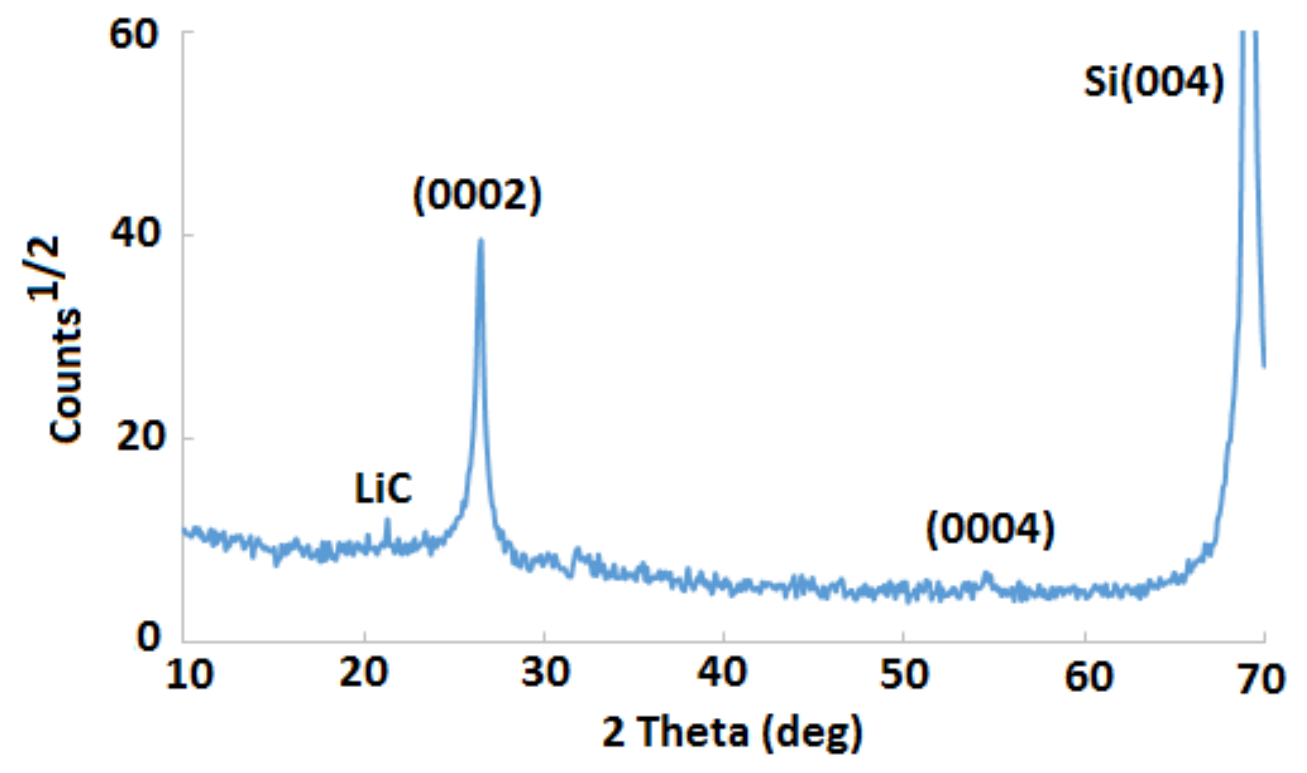

Figure 2(b). X-ray diffraction peak profile obtained from Li-graphene film on Si (100) substrate. The graphite peaks (0002) and (0004) are indexed. The small peak from LiC phase is not strong because both $\mathrm{Li}$ and $\mathrm{C}$ are elements with low atomic number and the fraction of LiC phase in the graphene film is very small. 

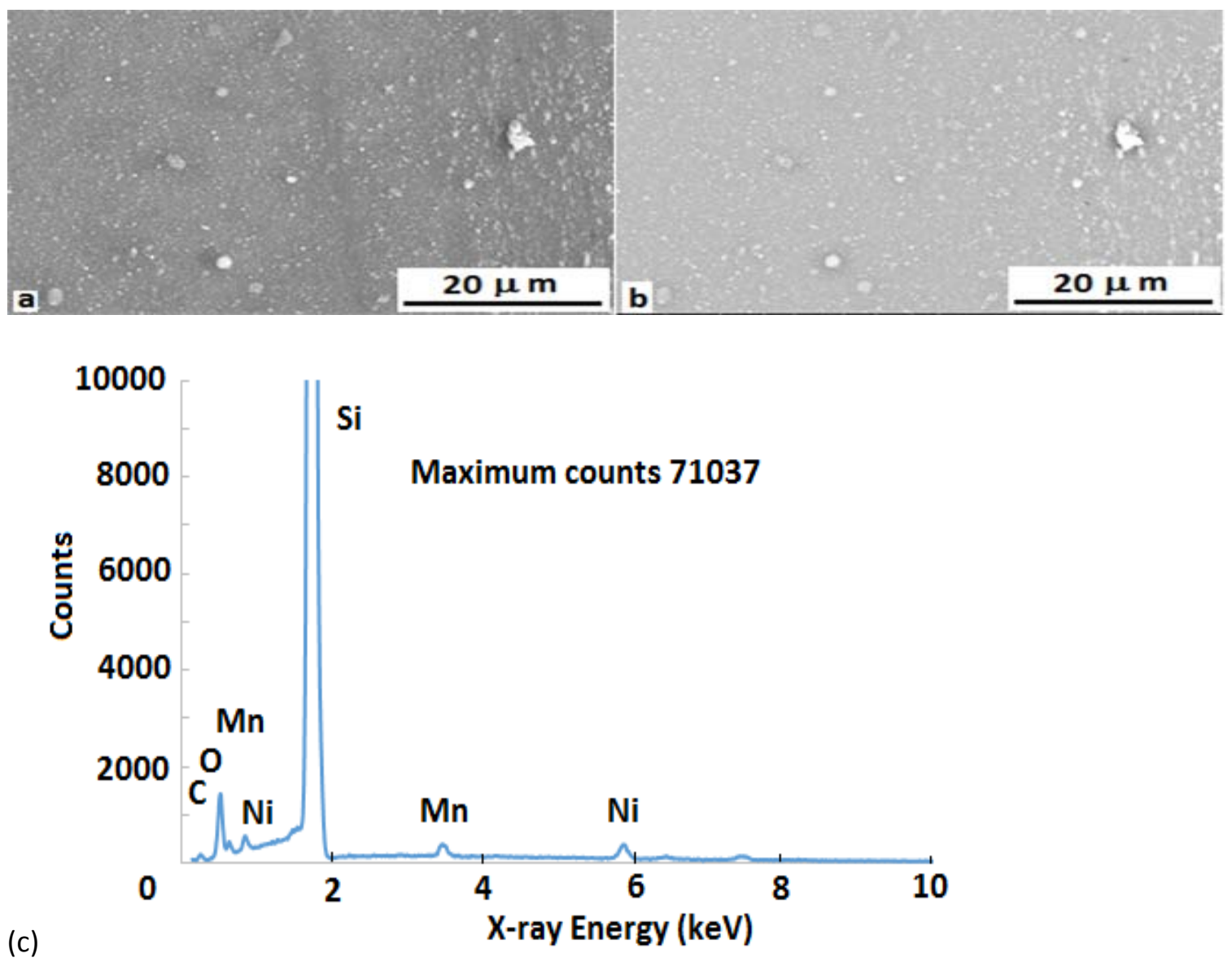

Figure 3. (a) Secondary electron image and (b) backscattering image of the LiNiMnO film on Si substrate deposited by LPVD. The thickness of the film is measured to be $0.4 \mu \mathrm{m}$. (c) Energy dispersive spectrum collected from the LiNiMnO film on Si showing the presence of $\mathrm{Ni}, \mathrm{Mn}$ and $\mathrm{O}$ in the film. The signal from $\mathrm{C}$ is thought to arise from hydrocarbons present on the surface in the SEM. 

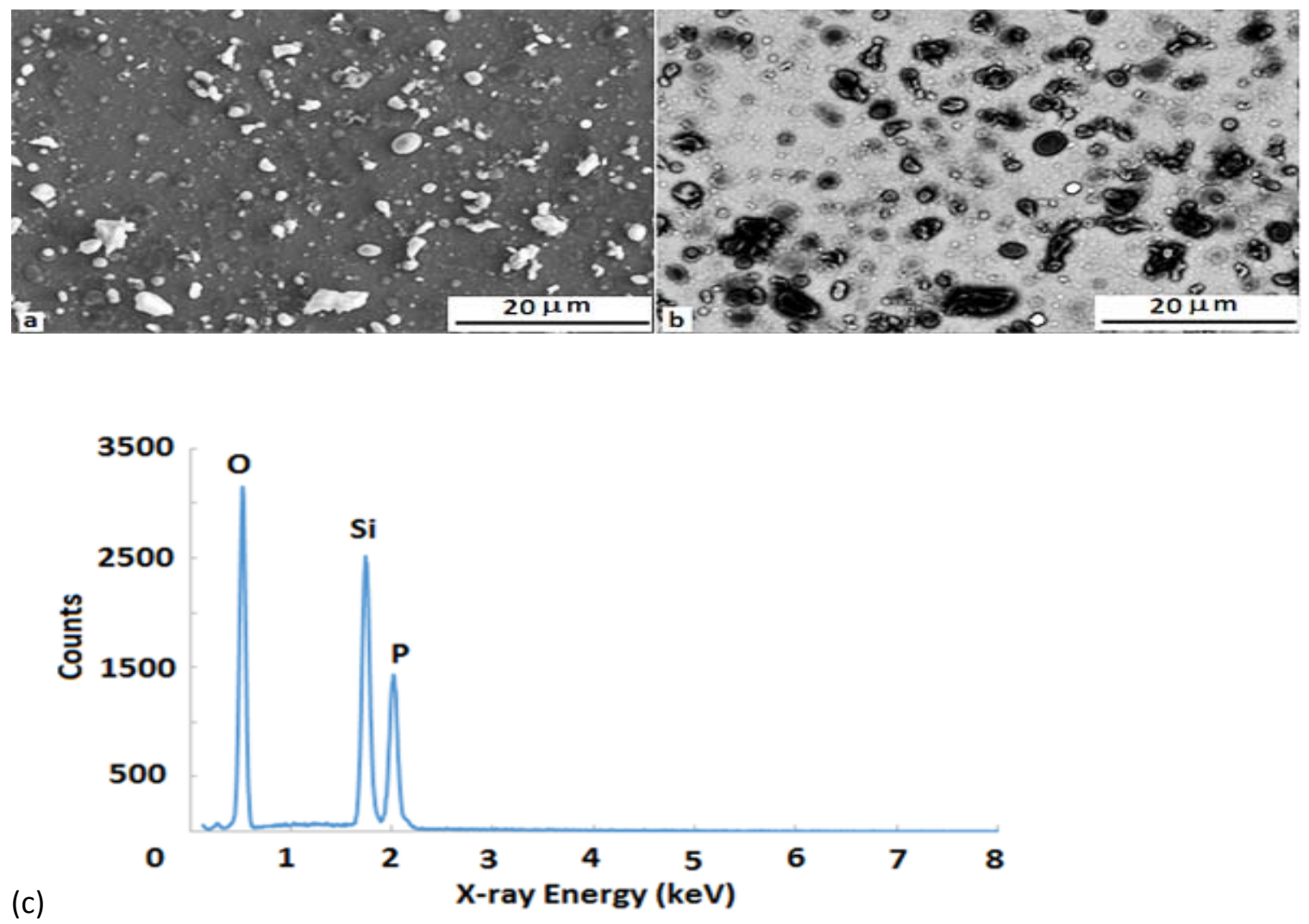

Figure 4. (a) Secondary electron image and (b) backscattering image of the LIPON film on Si substrate deposited by LPVD. The thickness of the film is measured to be $0.4 \mu \mathrm{m}$. (c) Energy dispersive spectrum collected from the LIPON film on Si showing the presence of $\mathrm{P}$ and $\mathrm{O}$ in the film. The signal from $\mathrm{N}$ is very weak. 

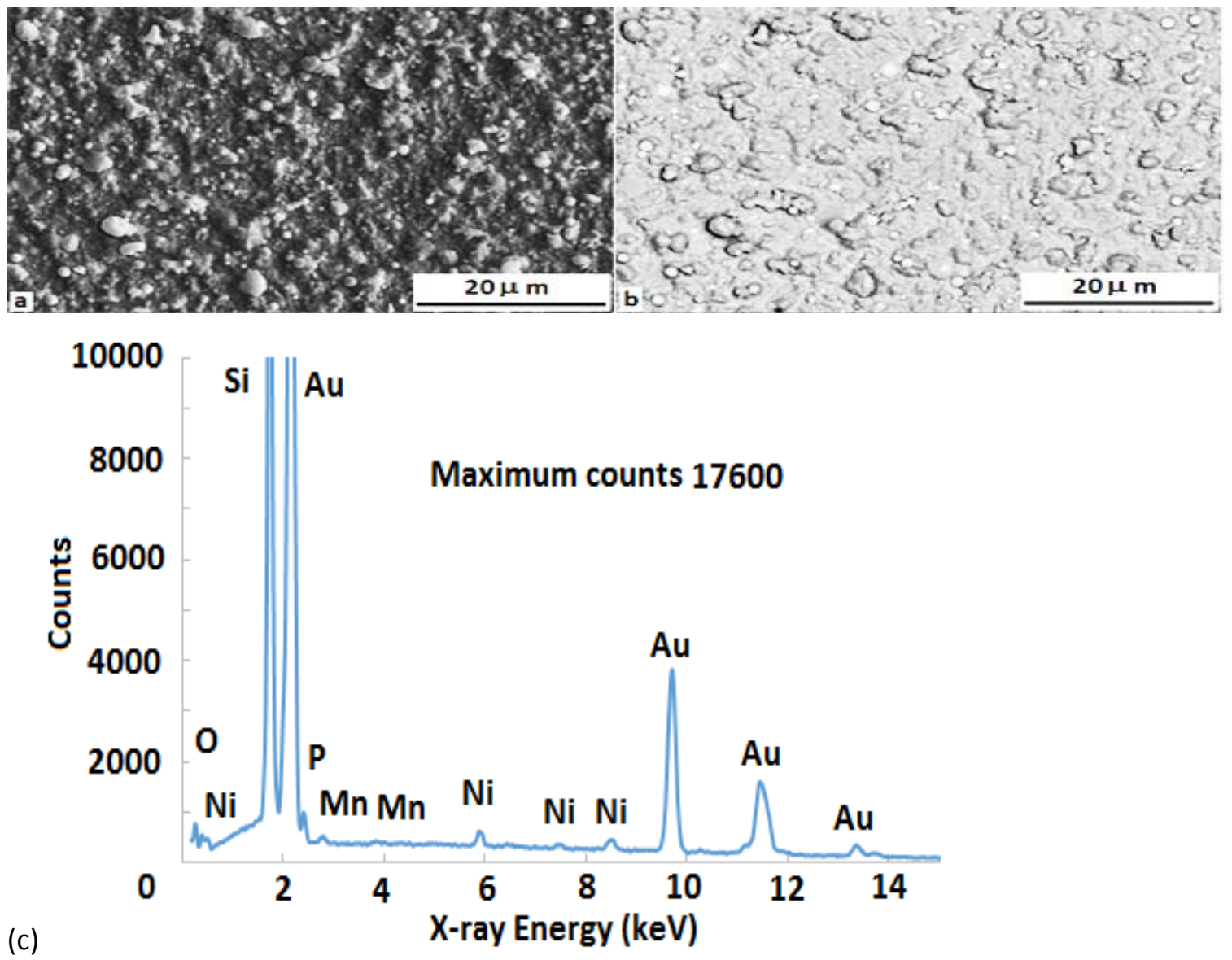

Figure 5. (a) Secondary electron image and (b) backscattering image of the LIPON-LiNiMnO film on Si substrate deposited by LPVD. The thickness of the LIPON film is $0.2 \mu \mathrm{m}$ and that of LiNiMnO film is 0.4 $\mu \mathrm{m}$. (c)Energy dispersive spectrum collected from the LIPON-LiNiMnO film on Si showing the presence of $\mathrm{Ni}, \mathrm{Mn}, \mathrm{P}$ and $\mathrm{O}$ in the film. The signal from $\mathrm{Au}$ is from coating given to obtain better image. Signal from $\mathrm{P}$ is between $\mathrm{Si}$ and Au signals and it is determined by the asymmetry of the Au peak. 

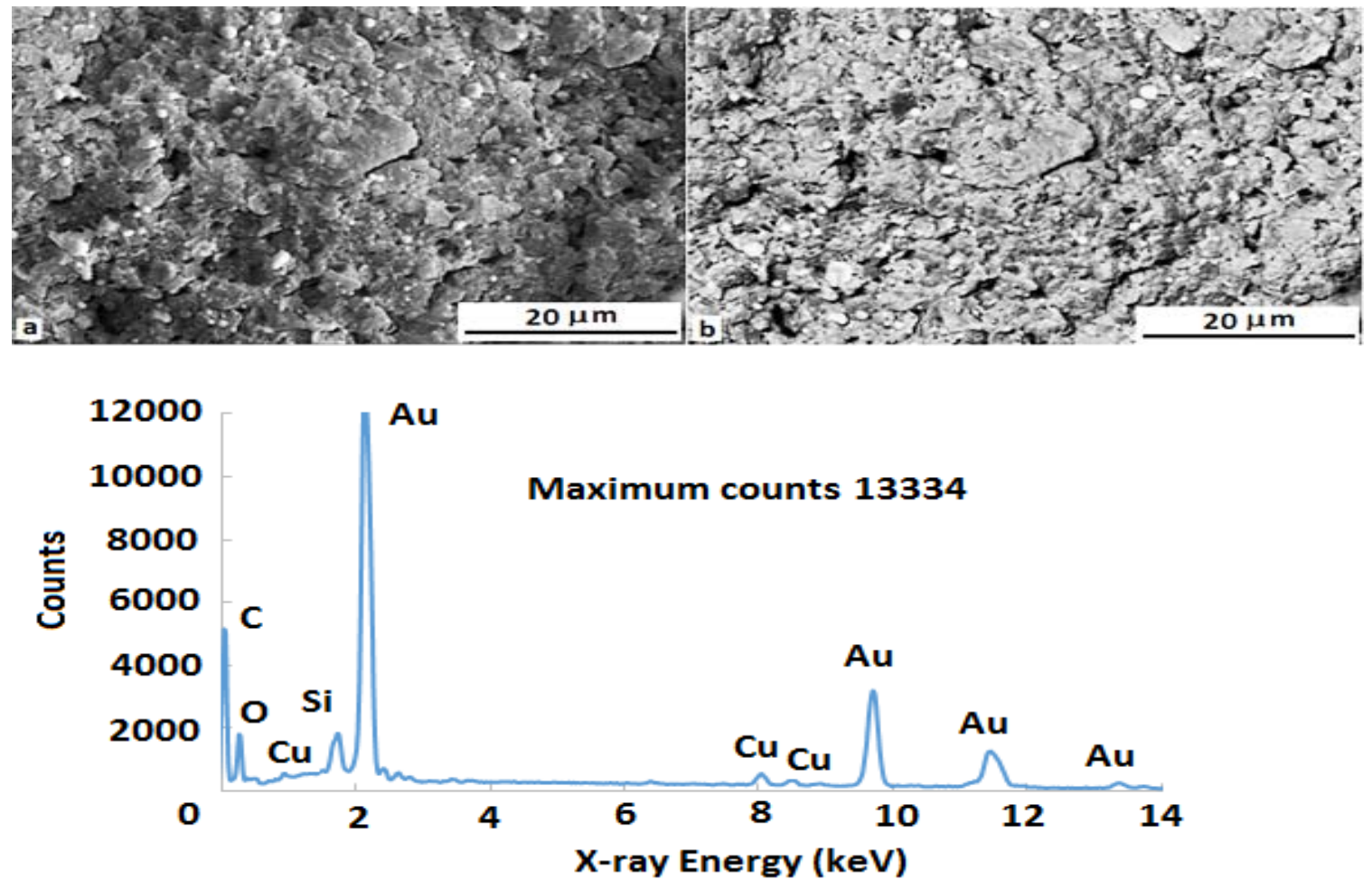

Figure 6. (a) Secondary electron image and (b) backscattering image of the Li-graphene film on Si substrate. Au film of thickness $0.15 \mu \mathrm{m}$ was deposited on the surface to getter a better image of the morphology of the graphene platelets. The thickness of the Li-graphene film is measured to be $4.0 \mu \mathrm{m}$. (c) Energy dispersive spectrum collected from the Li-graphene film on Si showing the presence of $\mathrm{C}$ and $\mathrm{O}$ in the film. The small signal from $\mathrm{Cu}$ is considered to arise from the $\mathrm{Li}$ foil that is dissolved in $\mathrm{HCl}$ to form the $\mathrm{LiCl}$. The $\mathrm{O}$ signal is from the $\mathrm{Li}_{2} \mathrm{O}$ formed during heating of the Li-graphene. 

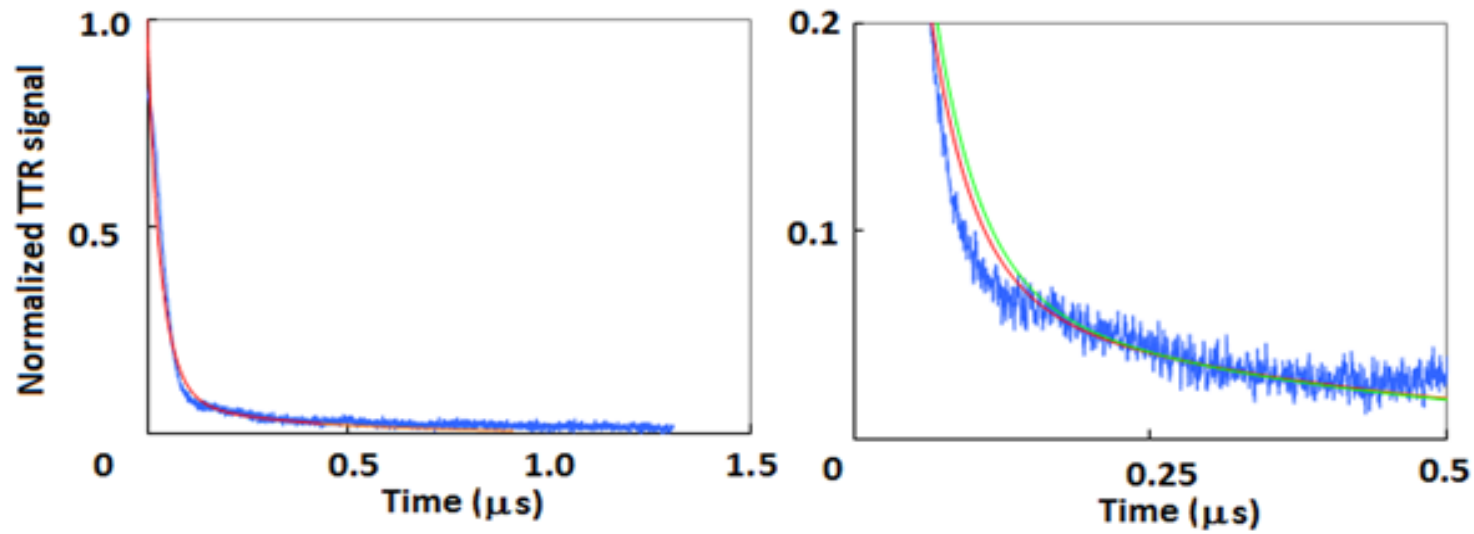

Figure 7(a). Normalized TTR signal from (a) the LiNiMnO film on Si.The blue line is the experimental result. The red line is the curve fitted result for values of parameters shown in table 1 . The inset on the right shows the details. The green line is obtained for $\mathrm{K}_{2}=30 \mathrm{Wm}^{-1} \mathrm{~K}^{-1}$ and $\mathrm{h}_{23}=150 \mathrm{MWm}^{-2} \mathrm{~K}^{-1}$ but with the same values for other parameters. 


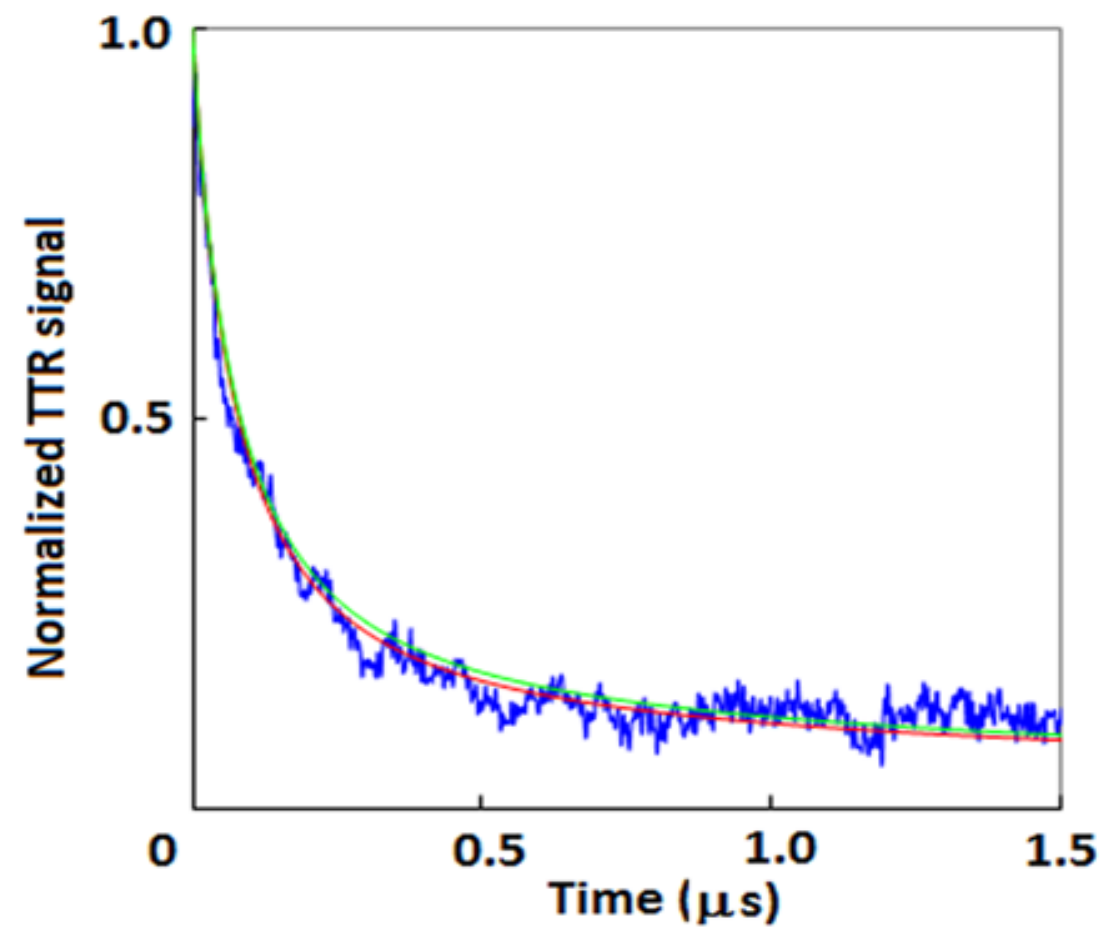

Figure 7(b). Normalized TTR signal fromthe LIPON film on Si. The blue line is the experimental result. The red line is the curve fitted result for values of parameters shown in table 1.The green line is obtained for $\mathrm{K}_{2}=6 \mathrm{Wm}^{-1} \mathrm{~K}^{-1}$ but with the same values for other parameters. 


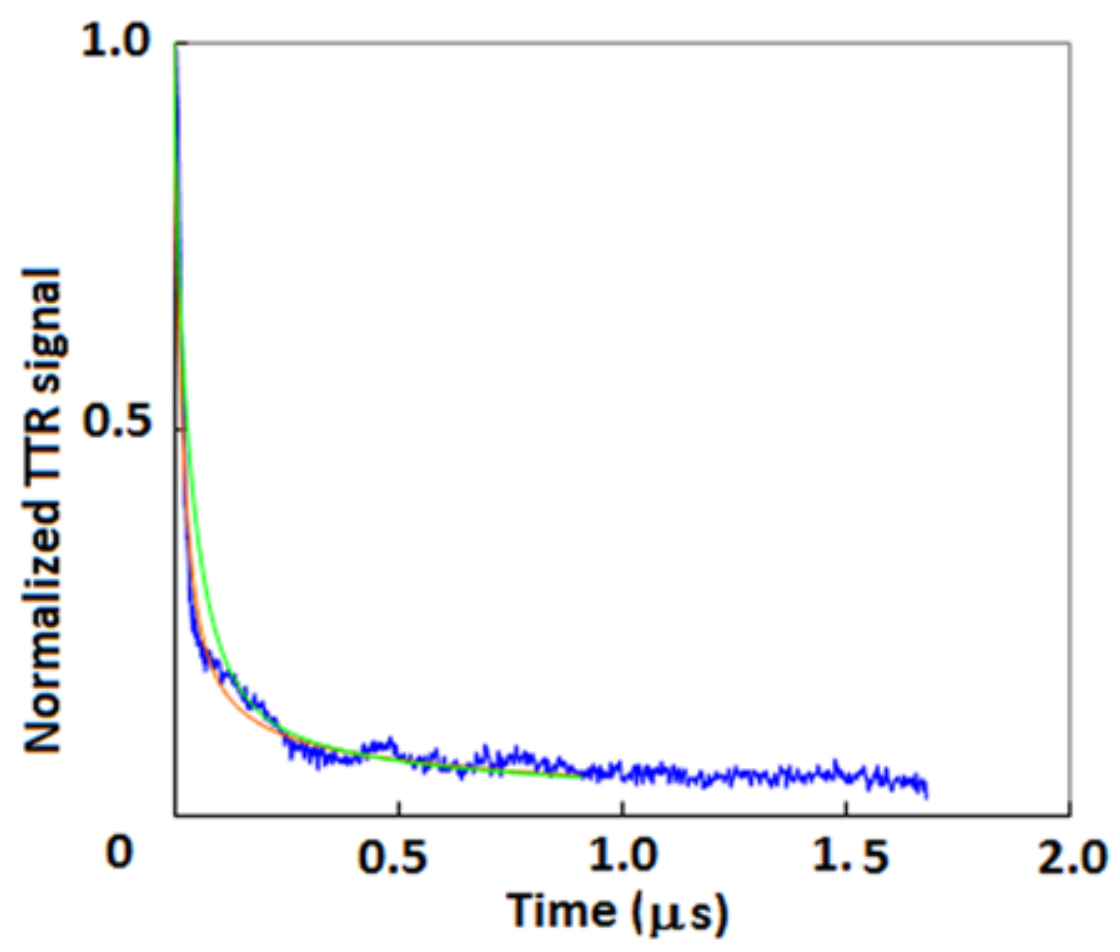

Figure $7(\mathrm{c})$. Normalized TTR signal fromthe LIPON film on LiNiMnO film on Si. The blue line is the experimental result. The red line is the curve fitted result for values of parameters shown in table 1.The green line is obtained for $\mathrm{K}_{2}=5 \mathrm{Wm}^{-1} \mathrm{~K}^{-1}$ but with the same values for other parameters. 


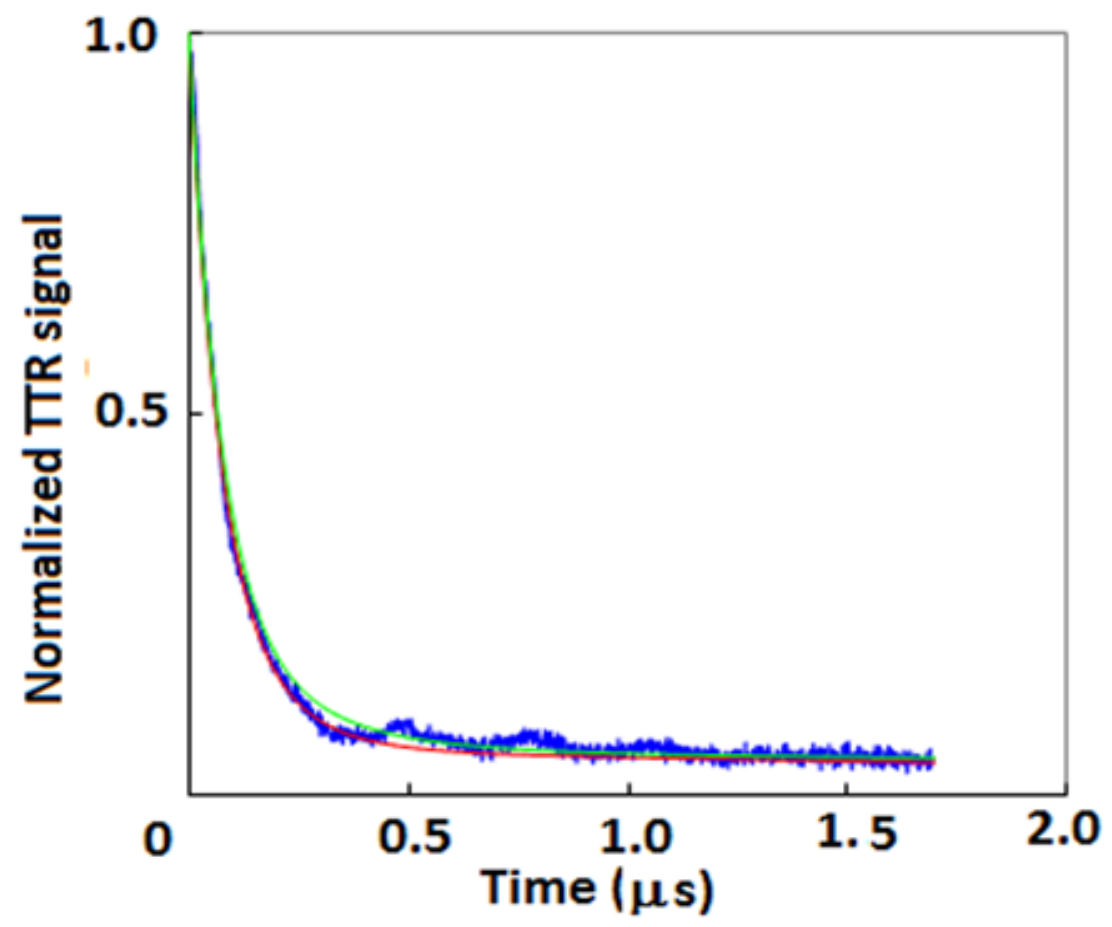

Figure $7(\mathrm{~d})$. Normalized TTR signal fromthe Li adsorbed graphene film on Si. The blue line is the experimental result. The red line is the curve fitted result for values of parameters shown in table 1.The green line is obtained for $K_{2}=30 \mathrm{Wm}^{-1} \mathrm{~K}^{-1}, \mathrm{~h}_{12}=\mathrm{h}_{23}=4 \mathrm{MWm}^{-2} \mathrm{~K}^{-1}$ but with the same values for other parameters. 


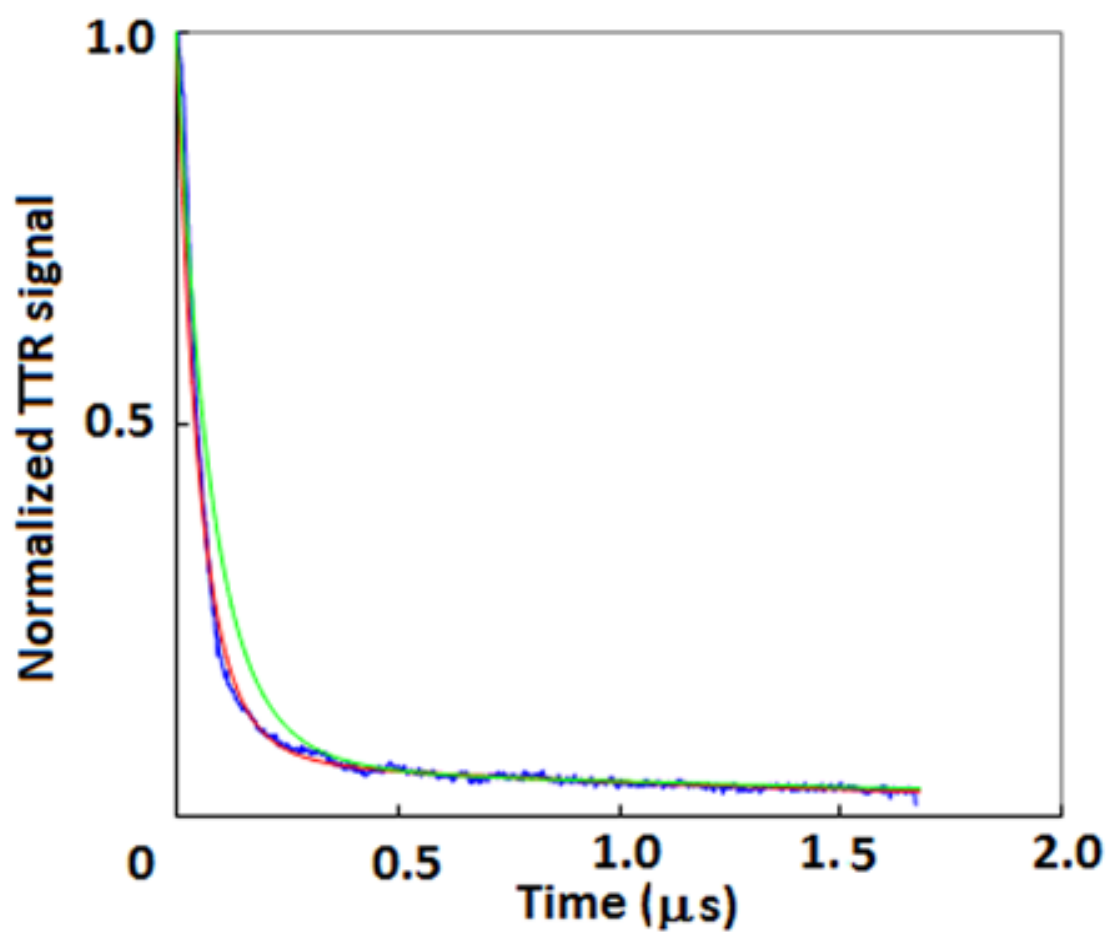

Figure 7(e). Normalized TTR signal from the graphene film on Si. The blue line is the experimental result. The red line is the curve fitted result for values of parameters shown in table 1.The green line is obtained for $\mathrm{K}_{2}=20 \mathrm{Wm}^{-1} \mathrm{~K}^{-1}$ and $\mathrm{h}_{23}=1 \mathrm{MWm}^{-2} \mathrm{~K}^{-1}$ but with the same values for other parameters. 